\title{
Advances in Crowd Analysis for Urban Applications through Urban Event Detection
}

\author{
M Shamim Kaiser, Senior Member, IEEE, Khin Lwin, Mufti Mahmud, Senior Member, IEEE, \\ Donya Hajializadeh, Tawee Chaipimonplin, Ahmed Sarhan, Member, IEEE, and M A Hossain, Member, IEEE
}

\begin{abstract}
The recent expansion of pervasive computing technology has contributed with novel means to pursue human activities in urban space. The urban dynamics unveiled by these means generate an enormous amount of data. These data are mainly endowed by portable and radio-frequency devices, transportation systems, video surveillance, satellites, unmanned aerial vehicles, and social networking services. This has opened a new avenue of opportunities, to understand and predict urban dynamics in detail, and plan various real-time services and applications in response to that. Over the last decade, certain aspects of the crowd, e.g. mobility, sentimental, size estimation and behavioral, have been analyzed in detail and the outcomes have been reported. This article mainly conducted an extensive survey on various data sources used for different urban applications, the stateof-the-art on urban data generation techniques and associated processing methods in order to demonstrate their merits and capabilities. Then, available open-access crowd datasets for urban event detection are provided along with relevant Application Programming Interfaces. In addition, an outlook on a support system for urban application is provided which fuses data from all the available pervasive technology sources and finally, some open challenges and promising research directions are outlined.
\end{abstract}

Index Terms-Urban sensing, pervasive technology, crowd mobility and management, information fusion, decision support system, benchmark datasets.

\section{INTRODUCTION}

All urban cities are becoming more interconnected with the recent developments in the information and communication technology (ICT) domain. Devices enabled with pervasive computing technologies, such as, smart cards [1], [2], wearable devices [3], Radio-Frequency (RF) communication devices (RFCD) (e.g., mobile phones [4], [5], [6], Bluetooth [7], [8],

Manuscript received March 6, 2017; revised August 15, 2017 and October 21, 2017; accepted November 2, 2017. This work was supported by the Erasmus MundusAction 2 Programme of the European Commission (SmartLink) under Contract 552077. The Associate Editor for this paper was P. Ye (Corresponding author: Mohammed Shamim Kaiser.)

M Shamim Kaiser is with the Institute of Information Technology, Jahangirnagar University, Dhaka, Bangladesh, e-mail: mskaiser@juniv.edu

Khin Lwin and M Alamgir Hossain are with Anglia Ruskin IT Research Institute of Anglia Ruskin University, UK, email: \{khin.lwin m.alamgir.hossain\}@anglia.ac.uk

M. Mahmud is with the NeuroChip Lab, Department of Biomedica Sciences, University of Padova, Via F. Marzolo 3, 35131 - Padova, Italy, e-mail: muftimahmud@gmail.com.

Donya Hajializadeh is with the Dept. of Engineering and the Built Environment, Anglia Ruskin University, UK, e-mail: donya.hajializadeh@anglia.ac.uk.

Ahmed Sarhan is with Faculty of Science and Technology, Anglia Ruskin University, e-mail: ahmed.sarhan@anglia.ac.uk

Tawee Chaipimonplin is with the Department of Geography, Chiang Mai University, e-mail: tawee.c@cmu.ac.th

Digital Object Identifier 10.1109/TITS.2017.2771746
[9], [10], Wireless Fidelity (WiFi) [11], [12], [13], RadioFrequency ID (RFID), and Global Positioning System (GPS) [14], [15], [16]), Optical-Wireless communication (OWC) (e.g., infrared or IR devices) [17], video surveillance [18], [19], [20], in conjunction with social media [21], [22], [23] and different event websites are part and parcel of our daily lives. Recent studies report that the above mentioned ubiquitous technologies can also be employed as sensors to collect data on human activities from the urban space and are uploaded to distributed / centralized databases. The acquired data are then preprocessed, mapped, and analyzed to comprehend and possibly predict the activity related aspects of urban life. However, the analyses of these activities are very challenging due to the inherent 'Big' and/or 'Fragmentary' nature of the data. Since the last decade, many works have been reported in the literature on their processing and development of various support systems (SS) targeting different urban applications (UA), e.g., real-time transportation operation and management, urban planning, food and water stock planning, optimal resource allocation, and crowd safety management [24], [25], [26].

Tailored mainly by the detected urban events, these SS mostly consist of two major components: an Urban Event Detection (UED) System or UEDS, and a Decision Support System or DSS. The UEDS detects a crowd event in a geo-location from the crowd data by monitoring changes in estimated crowd density with respect to a dynamically defined threshold value. After detecting an urban event, the required information are fed to the DSS to take necessary measure(s) as per the UA.

Currently the usage of pervasive technology (PT) has reached an unprecedented height. With the world population reaching 7.5 billion in 2017 [27], the PT sensors will generate huge amount of data. One of the major contributors to this data is the RF communication devices, out of which the dominant cellular phone subscribers is expected to be over $99.7 \%$ in 2017 [28]. The mobile activity data (also called call detail record (CDR) which logs data when users initiate telecommunication activities, e.g., placing / receiving a call, sending / receiving messages, and establishing Internet connection) in conjunction with the data provided by WiFi, GPS, and RFID render crowd information better than any other pervasive sources. In addition, video surveillance fitted in almost all infrastructures and peoples' wide usage of smart cards provide insights on crowd mobility. The social media networks and event websites contribute through crowd assembly data of public events at different geo-locations. Moreover, various 
government agencies use satellite and unmanned aerial vehicle (UAV) for surveillance which could be a possible source of crowd event data.

There have been diverse technologies and techniques to acquire as well as process this massive amount of data. Some related survey papers have been reported in the last few years. The techniques related to CDR data for Urban sensing applications were explored in [24]. Authors mentioned the pros and cons of CDR data, surveyed existing filtering and processing methods, and recommended the application of various datasets and methods for the different urban sensing applications. Jiang et al. reviewed methods for information extraction from triangulated CDR data, spatio-temporal analysis, and urban modeling [29]. Also, other studies reported real-time road traffic information extracted from the CDR data [30], [31], [32]. In [33], Janecek et al. proposed a novel approach combining signaling data ('idle' device information) together with CDR (SigCDR) to obtain canonical information of the mobile users in an area. They showed through vehicle tracking that there is a strong correlation between vehicles on the road and CDR with signaling. Schaue et al. employed $\mathrm{WiFi}$ and Bluetooth to estimate crowd densities and pedestrian flows [34]. However, the above mentioned studies dealt only with either CDR or SigCDR or RFCD data and did not take into account the local context information, crowd sentiment, and other sources of urban dynamics data. Junior et al. [35] and Zhan et al. [36] surveyed different crowd analysis techniques based on video surveillance data, but they left crowd counting and density estimation methods unexplored. In [37], Saleh et al. reviewed different crowd counting and density estimation methods from video data. In [38], Saif et al. presented sentiment analysis from status updates of Twitter to evaluate different organizations' individual performance, pointed out the limitation of different Twitter datasets used for sentiment analysis, and proposed STS-Gold dataset with sentiment labels. The social networks have been considered as a worthy source of information for research purposes due to their web-based application programming interfaces (APIs). In [39], Batrinca and Treleaven reviewed techniques, software tools, and platforms for social media analytics. Different data mining techniques and models for social media analysis were reported in [40], [41]. Gal-Tzur et al. [42] discussed different issues and challenges of social network's impact on transport services and related policy development. The usage of social network for transportation data collection, crowd modeling, and crowd size estimation have been previously reported [43], [44], [45], [46], [47], [26]. Ma et al. [48] introduced a distributed stream-based framework which fused various information to visualize data (at the different stacks) on a map. The framework detected the position of vehicle and then adjust the traffic load in real-time.

Though there exist few surveys on individual aspects of crowd data (i.e., data sources, processing methods, and event detection), yet, a comprehensive survey is missing which covers the state-of-the-art of various crowd data generation sources, existing processing methods for crowd estimation and crowd event detection, and the available UA that utilize the processed crowd data and their corresponding APIs. In addition, pros and cons of various crowd data (e.g., CDR, RFCD data from sources other than mobile phone, social network, etc.) in their usage with respective UA, are difficult to immediately identify. It is noteworthy that, with the existing approaches, information is usually extracted from one data source to plan appropriate UA which may provide partial knowledge about the crowd events leading to inaccuracy in the services provided by the UA. To improve the accuracy of such UA, fusion of extracted information from multiple sources are becoming increasingly essential. To mitigate this gap, this paper presents a comprehensive umbrella survey on the stateof-the-art in crowd analysis for UA through UED. It provides the advances in individual steps (i.e., crowd data sources and their generation, crowd data processing and analysis, and crowd estimation and UED) for designing adaptive and efficient UA from raw crowd data. Finally, also, a generic support system has been described, where, at first, extracted information coming from different data sources have been fused and required crowd information from the fused data have been extracted, then, an urban event has been detected. This event information then can be fed into appropriate decision support systems to design effective UA.

The survey of the literature was performed with the question to understand in detail the UED process from various crowd data for designing appropriate UA. To this goal, individual methodological articles connected to UED published during the period 1992 to June 2017 were identified through querying major article repositories (IEEE Xplore, Scopus, and Google Scholar) with specific search terms. These search terms included the keyword 'Crowd' and at least one of terms related to the analysis and application (e.g., data, analysis, social, mobilephone, estimation, urban, dynamics, detection, mobility, events, transport, and various ubiquitous urban sensors, etc.). The returned list of articles was carefully reviewed based on their relevance to the UED and the relevant articles have been included in the reference. Figure 1A shows a wordcloud (http://www.wordle.net/) of the articles' title words, where the size of a word denotes the relative number of occurrences in article titles. As seen in Figure $1 \mathrm{~B}$, these articles were then carefully assigned to research areas based on their publication titles as indicated by Thompson Reuters ${ }^{\mathrm{TM}}$ InCites ${ }^{\mathrm{TM}}{ }_{\text {Journal Citation Report }}{ }^{\mathrm{C}}$ (https://jcr. incites.thomsonreuters.com/) covering: Computer Science (including Personal/Ubiquitous/Mobile/Cloud Computing, Neurocomputing, Machine Learning, etc.), Transportation, Communication, Computer Vision, Multidisciplinary Sciences, Applications of Artificial Intelligence, and Others (including Civil/Electrical Engineering, Geography, Management). The main focus of these articles were the methods that describe detection of crowd dynamics provided by crowd data. These dynamics generally consist of crowd count [6], [7], [11], [13], [49], [50], [17], [51], [52], crowd trajectories/mobility [32], [53], [9], [14], [54], crowd sentiment [21], and crowd behavior [18], [20], [55], [56], [57], [58], [51], [59], [60]. These articles clearly showed an increasing trend in the crowd event detection research for UA (see Figure 1C). As the main purpose of this work has been to report latest progress in UED which depends on the crowd dynamics except for crowd behavior 

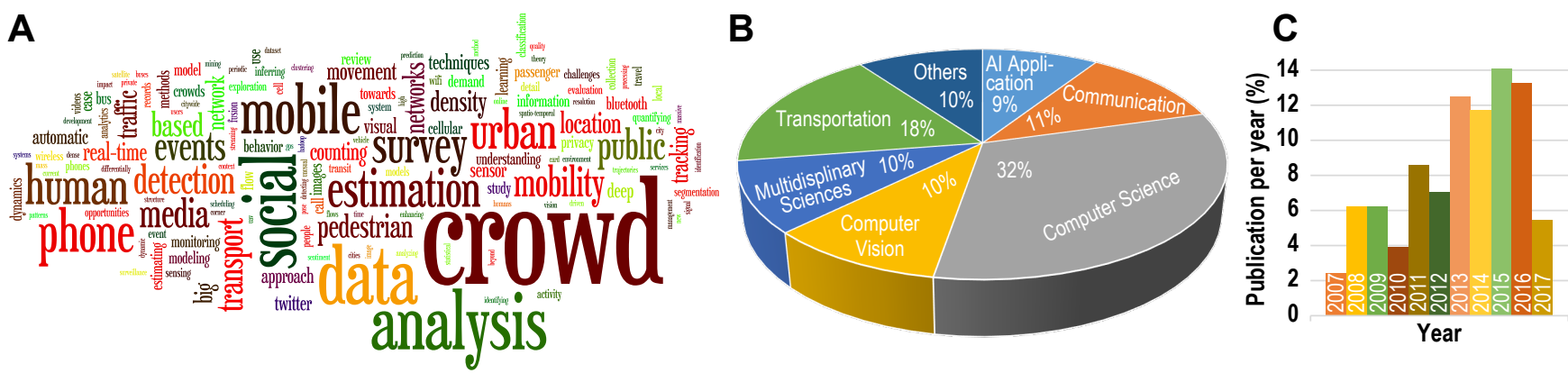

Fig. 1. Literature survey methodology depicted as retrieved keywords from article title (A), category wise publication distribution (B), and year wise publications in percentage $(\mathrm{C})$.

\section{Urban Space}

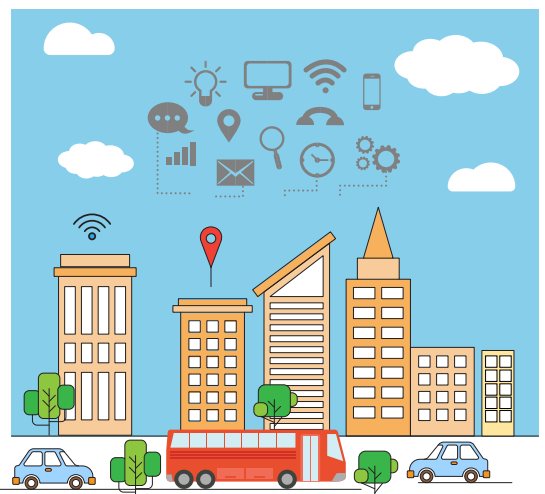

Support System

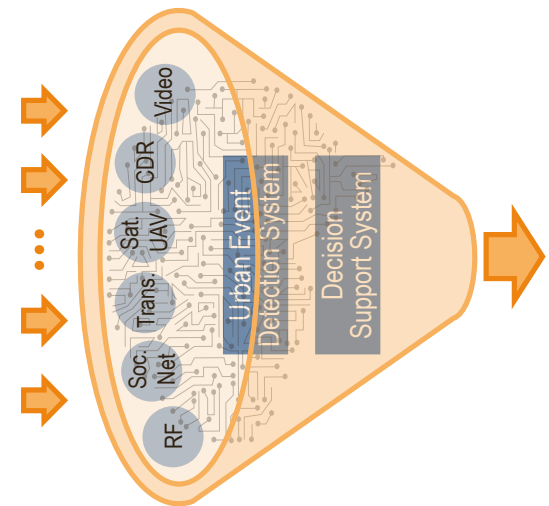

Urban Applications
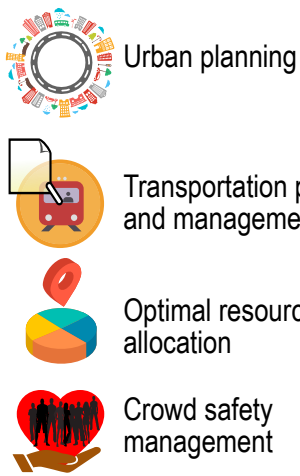

Transportation planning and management

Optimal resource

allocation

Crowd safety

management

Fig. 2. Smart urbanization through ubiquitous sensing. A modernized urban space facilitates its users with infrastructures to use a number of smart tools. The raw data generated by these ubiquitous sensors can be categorized based on their sources as: CDR data, RF based data, IR data, video data, transportation data, social network and event website data, and satellite and UAV data. The information coming from various ubiquitous sensors are fused and fed into intelligent processing systems (e.g., UEDS). The knowledge provided by the UEDS from the fused information, as well as local context, the crowd size and probable urban event at a geo-location are predicted. This knowledge can further be utilized in personalizing and providing various urban applications using application specific DSSs.

(also detectable mainly using video data), the studies related to crowd behavior has not been reported here.

The main contributions of the paper can be categorized as follows:

- Review on various crowd data for different UA;

- Survey on these data generation and processing methods for crowd event detection;

- Study on available open access benchmark crowd datasets and different APIs;

- Describe a generic framework in the form of Urban Event Detection System to demonstrate the possible usage of the crowd data; and

- Identify some open challenges, and future research directions.

The organization of this paper is as follows: section II narrates an overview of the urban sensing applications and different crowd data sources used for various UA. Section III discusses the existing state-of-the-art crowd analysis techniques, including information fusion, for crowd size estimation and crowd event detection. Section IV presents some available APIs and benchmark datasets used for crowd size estimation. The exemplary and generic framework for the SS has been outlined in Section V. Section VI lists some open challenges and probable research opportunities in crowd event detection. The article concludes in Section VII.

\section{OVERVIEW OF URbAN SENSING AND VARIOUS CROWD DATA SOURCES}

\section{A. Crowd Mobility and Urban Sensing}

Crowd is, "... a large group of individuals in the same physical environment, sharing a mutual interest" [61] which is, defined by the shared emotional experiences among the members [62]. Also, structurally, few individuals together form a group, and several of these groups with a common goal make them a crowd. The management of crowd is very challenging and a lot of research have been conducted since the last decade related to crowd count, crowd density estimation, crowd pattern, and crowd event estimation. The mobility of a crowd's members in the urban space for various purposes (e.g., commuting, shopping, traveling, activities, etc.) make them interact with the environment (e.g., purchase a ticket, activity using mobile phone, appearance in a social network, etc.) which generate huge amount of data. These data includemobile activity data, RF data, IR data, transport data (including ticket and parking lot), social network data, video surveillance data, satellite image, UAV, and census data (See figure 2). 

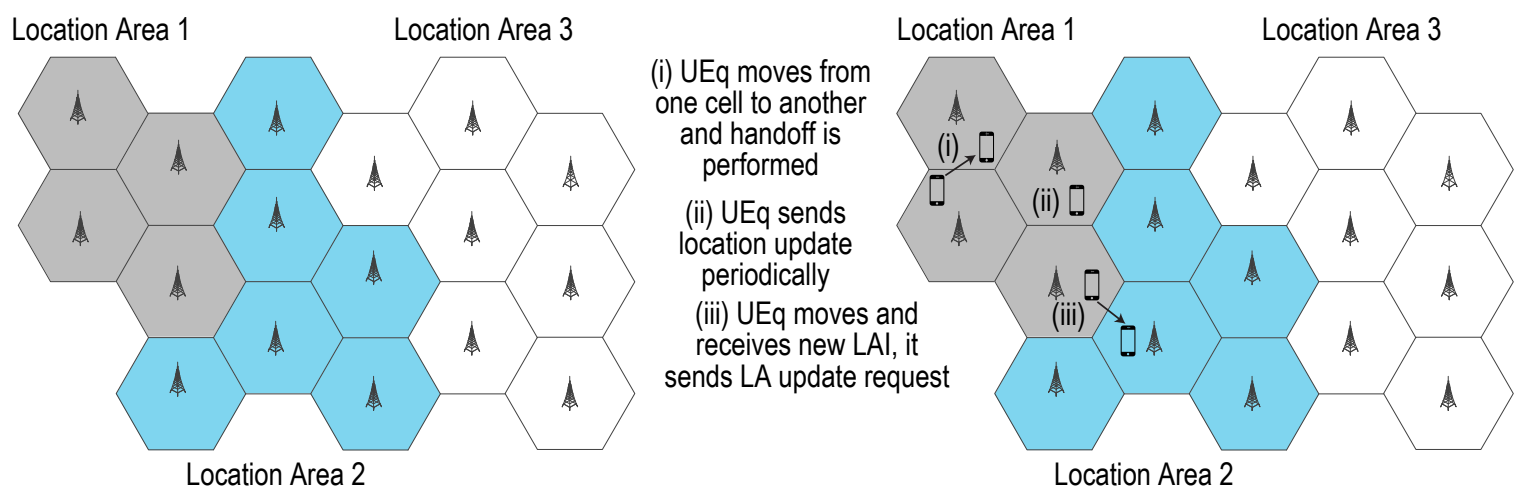

Fig. 3. Location areas and BSs of a cellular network (Left). The cells are divided into groups called Location/Routing area; LAI are updated during three scenarios (Right) - (i) UEq moves from one cell to new cell and handoff is performed, (ii) UEq sends location update periodically, and (iii) UEq moves and receives new LAI, it sends LA update request. Different methods of LAI updates of a UEq are illustrated.

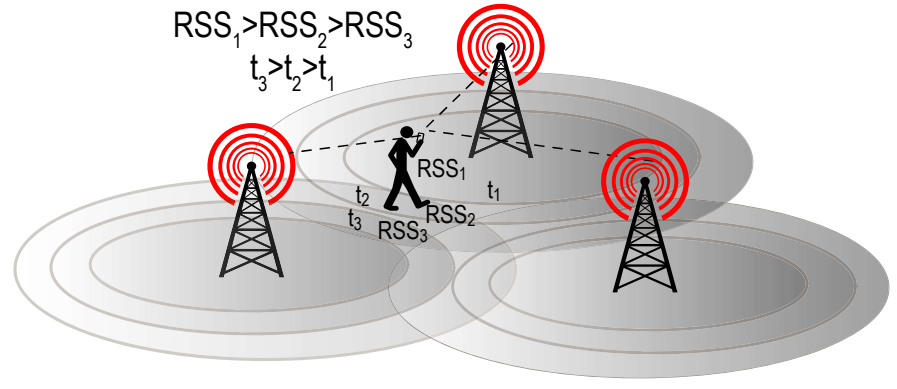

Fig. 4. Triangulation principle confirms the location of an UEq. Based on RSS value and TA $\left(t_{i}\right)$, BSs identifies a UEq with high accuracy.

These heterogeneous crowd data produced by different urban sensors in the urban space are collected, fused, and analyzed to estimate the activities, and mobility of urban inhabitants through a process called Urban Event Detection. Also, careful analysis of these data may provide useful information about the crowd, such as, crowd size prediction, crowd monitoring, and most importantly- 'urban application planning'. For example, among the urban services, transportation is a vital service and is directly related to the mobility of the crowd. The UEDS estimates the crowd size and detects a crowd event in a geolocation if the changes in crowd density estimated from the crowd data collected by the different urban sensors are higher compared to a given threshold value. Here Census data may be used as baseline population information at a given geolocation

Table I lists the various s, and the data source(s) used in those applications. It can be noticed that data coming from almost all the sources support 'event detection' process. In addition, most reported articles did not use more than 1 sources (at times maximum 2) for their event detection or decision making process.

\section{B. Crowd Data-Sources, Generation, and Applications}

This section provides overview of various crowd data sources, generation of such data at the sources, and their possible urban applications.
1) Mobile Phone Network Data: The CDR data is generated when voice/text are exchanged between user equipments (UEq) and each of these records contain the source user ID $\left(S_{I D}\right)$, destination user ID $\left(D_{I D}\right)$, source cell ID $\left(S C_{I D}\right)$, destination cell ID $\left(D C_{I D}\right)$, timestamp $(T S)$, call duration $(t)$, etc. as shown in Table II. The cell ID includes the latitude and longitude information of a geo-location (Table II). The Internet protocol detail record (IPDR) also provides a significant amount of information about user's activities. Some fields of IPDR are Source and destination IP addresses, service type, vendor ID, type of application, total upstream/downstream data volume, etc. In both cases, the UEq is communicating with the Cellular Access Point or CAP (e.g., Base station or BS). The area covered by a CAP or BS is known as a 'Cell' (Figure 3 (Left)). A geographical area is divided into a large number of cells. The cell size is not uniform, and depends on many factors like propagation environment and system condition. A single base station controller (BSC) controls 10 to $100 \mathrm{BSs}$. Measurement of Received signal strength (RSS) from UEq, and handover control are the core functions of the BSC. A Mobile Switching Center (MSC) controls all BSCs. A group of cells are called a location area or routing area or tracking area. When an UEq is idle and within the location area, it is reached via a periodic paging signal. If the UEq moves to the different location area, it executes location update. When an UEq is moved to another cell during a call or data session, handover is performed and location area of the UEq is updated. Figure 3 (right) shows that the location area information (LAI) is updated:

- when handover (such as both vertical (e.g., 3G to 4G), and horizontal handover (e.g., one BS to another BS)) occurs [63];

- periodically the current location of UEq in a BS;

- when an UEq moves between adjacent location areas.

The cellular system uses triangulation principle to identify an UEq in a geo-location (see Figure 4). In this principle, all three BSs use timing advance (TA) and RSS to identify the location of an UEq with relatively high accuracy. As these mobility data contain user's privacy information, cellular service providers share a portion of these data after using an anonymity technique based on the rules and restrictions of the 
TABLE I

DATA SOURCE FOR DiFFERENT URBAN APPLICATIONS

\begin{tabular}{|c|c|c|c|c|c|c|c|c|c|c|c|c|}
\hline Urb. Sen. Param. & CDR & Orig.-Dest. Trans. & GPS & Bluetooth & WiFi & RFID & Video & Soc. Net. & Act.Trac. & Sat. & UAV & Census \\
\hline Land Use & $\checkmark$ & $\checkmark$ & & & & & & & & $\checkmark$ & $\checkmark$ & $\checkmark$ \\
\hline Mobility pattern & $\sqrt{ }$ & $\sqrt{ }$ & $\sqrt{ }$ & & & & & & & & $\sqrt{ }$ & \\
\hline Periodic activity & $\checkmark$ & $\checkmark$ & $\checkmark$ & $\checkmark$ & $\checkmark$ & $\checkmark$ & & $\checkmark$ & $\checkmark$ & $\sqrt{ }$ & $\sqrt{ }$ & \\
\hline Social tier & $\sqrt{ }$ & & & & & & & $\sqrt{ }$ & & & & \\
\hline Crowd Sentiment & & & & & & & & $\checkmark$ & & & & \\
\hline Crowd behavior & & & & & & & $\sqrt{ }$ & & & & $\sqrt{ }$ & \\
\hline Crowd count & $\sqrt{ }$ & $\sqrt{ }$ & $\sqrt{ }$ & $\sqrt{ }$ & $\sqrt{ }$ & $\sqrt{ }$ & $\sqrt{ }$ & $\sqrt{ }$ & $\sqrt{ }$ & $\sqrt{ }$ & $\sqrt{ }$ & \\
\hline Pop. density & $\sqrt{ }$ & $\sqrt{ }$ & $\checkmark$ & $\sqrt{ }$ & $\sqrt{ }$ & $\sqrt{ }$ & & & & $\sqrt{ }$ & $\sqrt{ }$ & $\checkmark$ \\
\hline Event detection & $\sqrt{ }$ & $\sqrt{ }$ & $\sqrt{ }$ & $\sqrt{ }$ & $\sqrt{ }$ & $\sqrt{ }$ & $\sqrt{ }$ & $\sqrt{ }$ & $\sqrt{ }$ & $\sqrt{ }$ & $\sqrt{ }$ & \\
\hline
\end{tabular}

Legends: Urb. Sen. Param.-Urban Sensing Parameter; Orig.-Dest. Trans.-Origin-Destination Matrix for Transportation; Soc. Net.-Social Network; Act. Trac.-Activity Tracker; Sat.-Satellite;

country. Real-time aggregated data can be difficult to access. Thus a possible solution is that a subset of this data can be analyzed.

The CDR/IPDR data are event-driven, and captured when cellular mobile activities such as voice calls, text messages, and data usages (i.e. 3G/4G/5G) are detected. Such data offer some opportunities for different services as it reflects the spatio-temporal patterns of the users' activities. These data have been used for trip analysis [25], [64], [65], detecting social events [66], urban sensing [24], city modeling [29], finding crowd trajectories [32], planning and modeling urban transport [65], [67], [68], [69], estimating an actual crowd size in an event [49], [53], detecting tourist spot [70], and so on.

TABLE II

CDR CONTENT

\begin{tabular}{|c|c|c|c|c|c|c|}
\hline$S_{I D}$ & $D_{I D}$ & $\begin{array}{c}S C_{I D} \\
(\text { Lat }, \text { Long })\end{array}$ & $\begin{array}{c}D C_{I D} \\
(\text { Lat }, \text { Long })\end{array}$ & $T S$ & $t$ & $\begin{array}{c}\text { Other } \\
\text { Fields }\end{array}$ \\
\hline
\end{tabular}

2) RF and IR Based Data: RF data can be used to obtain important information about the crowd size and mobility. Almost all the smartphone devices are equipped with Bluetooth, WiFi, GPS and other RF sensors.

a) WiFi and Bluetooth Data: RF access point scans UEq, such as smartphones or other RF devices, with high directional RF beams. In response to the scan, UEq sends some information (such as MAC address of the wireless interface, RSS indicator, the vendor of the smartphone, RF access point information, class of devices in case of Bluetooth, timestamp, etc.) to the RF access point. Based on the information received, the RF access point detects the user's geo-location at a particular timestamp, then remove the redundant entries (due to multiple switch-on interfaces) from the database as one entry per device can exist for a specific space and time. The stationary $\mathrm{WiFi}$ and Bluetooth scanners with highly directional antennas can be placed at specific locations to gather data about crowd dynamics in a large scale crowd event. The WiFi and Bluetooth data can be used to monitor crowd size and mobility [6], [7], [9], [11], [13], [50], [93], [94], retrieving vehicle trajectories [10], [73], and crowd density estimation [6]. b) GPS Data: GPS sensors receive signals from at least four satellites which are visible at that time. Each satellite transmits spatio-temporal information to the GPS sensor at regular intervals. The GPS receiver reads the information provided by the satellites and determines its geo-location. The geo-location data generated by the GPS sensor are mainly used for crowd tracking [14], [90].

c) RFID Data: A RFID reader (also known as an Interrogator) reads the RFID tags (or smart labels) by using RF beams. RFID tags also contain an integrated circuit with an antenna. The information read from the tag is transferred to a host computer for storing and analyzing. This RFID data can also be used to derive crowd information (e.g., crowd counting and monitoring) [95], [93].

d) OWC Data: Optical wireless communications (OWC) employs free space as communication media to transmit (in)visible light (e.g., infrared or IR) to carry the signal between the transmitter-receiver pair(s) e.g., light emitting diode- photodiode/phototransistor pairs. In contrast to RF signal, the optical count/data transmission is ultra fast and more accurate. But the coverage area is small due to ambient condition. The OWC system data can be used to derive crowd counting information [17].

3) Transport Data: Nowadays, many services are interconnected through the Internet and ICT infrastructures. The umbrella term 'transport data' is used to denote data that are generated by several subsources and/or sensors including fare cards, smart card, public bicycle systems, parking occupancy system, etc. People use these means for transit fare payment, parking fee payment, and ticket booking via web-based applications (apps) or mobile apps. Data generated by these means in addition to the passenger's ticket purchase, booking, and vehicle movements information (e.g., arrival time, service time, delay time, etc.) are transferred to the central system and stored in the transportation database in almost real-time. A detailed review on smart card from the perspective of data generation, processing, and applications has been reported previously [1]. These data contain different information and insights about the transport services and opinions of the passengers. The parking lot occupancy information is also essential to identify a crowd event near it.

The raw transportation data are converted to origin-to- 
TABLE III

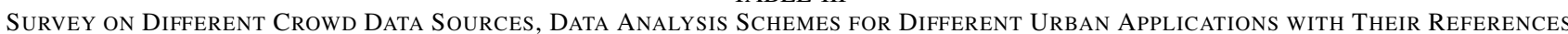

\begin{tabular}{|c|c|c|c|}
\hline Data Source & Data Analysis Scheme & Applications & Ref. \\
\hline \multirow{11}{*}{ CDR } & A persistence probability is used to identify user at a specific BS & Est. Cr. Sz. & [49] \\
\hline & Find the spatial and temporal regularity in daily human mobility & Cr. Trj. / Cr. Sz. & [32] \\
\hline & Find the O-D matrices for the transportation & Urb. Trn. Pln. & [71] \\
\hline & A method is used to detect the daily mobility patterns of large-scale crowd & Cr. Trj. / Cr. Sz. & {$[53]$} \\
\hline & A SSL based framework that identified individuals' trips & Trip analysis & [25] \\
\hline & Tools to identify and validate road usage patterns from O-D matrices & Trip analysis & [64] \\
\hline & A tool to identify clusters of cell towers with identical call patterns & Urb. Trn. Pln. & [66] \\
\hline & Find relation between mobility and workload dynamics due to large events & Management of UAs & {$[72]$} \\
\hline & Find the frequency of travel back and forth between LA and NY & Urb. Trn. Pln. & {$[65]$} \\
\hline & Pin down the city's hotspot \& spatial structure at particular hours of a day & Urb. Trn. Pln. & [67], [68] \\
\hline & Optimized a transit network based on mobility patterns of people & Urb. Trn. Pln. & [69] \\
\hline \multirow{8}{*}{ RF/IR } & A framework to monitor a crowd with WiFi activated handheld devices & Est. Cr. Sz. & {$[11],[13]$} \\
\hline & A framework to monitor a crowd with Bluetooth activated handheld devices & Est. Cr. Sz. & [6], [7], [11] \\
\hline & Bluetooth scan analyzed the crowd trajectory in a mass gathering & Cr. Trj. & {$[9]$} \\
\hline & Bluetooth scan was employed to analyze O-D Martics & Urb. Trn. Pln. & [73] \\
\hline & GPS data can be utilized to perceive the pedestrian motion & Cr. Trj. / Cr. Sz. & [14] \\
\hline & A framework to monitor a crowd with RFID & Est. Cr. Sz. & {$[50]$} \\
\hline & A procedure to extricate vehicular trajectories and trip information & vehicular trajectories & {$[10]$} \\
\hline & IR based based Pedestrian count using Non-parametric statistical method & Cr. Co. & [17] \\
\hline \multirow{5}{*}{ Transport } & A model was used for the timing of trip based on set of ana. tras. serv. plan & Cr co. UED & [74] \\
\hline & A heuristic algorithm analyzes O-D matrix & Est. trav. Mob. & {$[2]$} \\
\hline & A probabilistic model analyzes smart card data & Est. Cr. Sz. \& UED & {$[51]$} \\
\hline & A framework inspects Bicycle mobility data & Urb. Att.\& Urb. Mod. & [75] \\
\hline & MNL and EC models observe different aspects of crowding on travel time & Est. Cr. Sz.\& UED & [52] \\
\hline \multirow{6}{*}{$\mathrm{SN}$} & DBSCAN algorithm is employed to spatio-temporal tweets & Est. Cr. Sz. \& UED. & {$[76]$} \\
\hline & A framework analyzes tweets related to sentiment of passenger & Urb. Trn. Pln. & [21] \\
\hline & An algorithm analyzes Tweets & UED & {$[23]$} \\
\hline & BDP analyzes geo-tagged SN data and illustrates events on GIS & RT UED & [77] \\
\hline & Combines DBC, SN data mining and outlier detection to detect crowds & RT UED & [78] \\
\hline & The MABED model analyzes tweets of each event & Vis. To. Tr. Cr. Ev. & [79] \\
\hline \multirow{11}{*}{ Video Data } & Regression methods dealing with many co-linearity among features. & image-based Cr. Co. & [80] \\
\hline & Sta. cr-gr anal. to estimate the stationary time of foreground pixels & Trf. Fl. Co. & [81] \\
\hline & Deep CNN was used to extract extract high and low level features & Est. Cr. Co. Den. & [82] \\
\hline & A model is used to segment and detect individual & Est. Cr. Co. & [37] \\
\hline & Each independent motions are detected using clustering & Est. Cr. Co. & [54] \\
\hline & A linear regression model was used foreground pixels as well as edge & Est. Cr. Co. Den. & [83] \\
\hline & A FFNN method was employed foreground pixels as well as edge & Est. Cr. Co. Den. & {$[84]$} \\
\hline & A LR model and foreground pixel employed as an image feature & Est. Cr. Co. Den. & [85] \\
\hline & A BPNN and foreground segmentation as well as edge detection were used & Est. Cr. Co. Den. & {$[86]$} \\
\hline & Texture-based analysis was used & Est. Cr. Co. Den. & [37] \\
\hline & Interest points were employed as feature masked with optical flow model & Est. Cr. Co. Den. & [87] \\
\hline \multirow{2}{*}{ Satellite \& UAV } & An algorithm for different resolution images using probabilistic thresholds & Est. Cr. Co. Den. & [88] \\
\hline & Tex. clas. met. to identify crowded regions from aerial images & Est. Cr. Co. Den. & [89] \\
\hline \multirow{5}{*}{ Hybrid } & App and GPS trajectory data were fused & Est. Cr. Co. Den. & [90] \\
\hline & CDR/IPDR and Twitter data were fused & Est.Cr. Sz. \& UED & {$[22]$} \\
\hline & CDR and Flicker data were fused & Urb. att. & [70] \\
\hline & Twitter and Instagram Data were fused & Det. on-going evt. & {$[91]$} \\
\hline & Bluetooth and GPS location Data were fused and analyzed & Trk. Cr. Act./Cr. Trj. & [92] \\
\hline
\end{tabular}

Legends: Est. Cr. Sz.: Estimate Crowd size; Cr. Trj.: Crowd trajectory; Cr. Sz.: Crowd size; Urb. Trn. Pln.: Urban Transport planning; SSL-Supervised Statistical Learning; Cr. Co.: Crowd count; Est. trav. mob. -Estimated travelers mobility; Urb. Att.- Find Urban Attractiveness; Urb. Mod.-Urban modeling; BDP-Big data platform; RT UED- Real-time urban event detection; Vis. To Tr Cr Ev- Visualization tool to track crowd event ; Trf. Fl. Co.: Traffic Flow Counts; CNNConvolutional Neural Network; Est. Cr. Co. Den. - Estimate crowd count and crowd density; LR- linear regression model; FFNN-

feed-forward neural network; BPNN-back propagation neural network; Tex. Clas. Met. Texture classification methods; Det. on-going evt.-Detected on-going event; Trk. Cr. Act. -Track crowd activity

destination (O-D) matrix for using them in different UA. These data are mainly used in transportation planning and management [74], [52], [51], crowd density and crowd event estimation [51], traveler trajectory and mobility pattern estimation [2], [75], point of interest identification [96], etc. Also, automatic vehicle location data can be used for transportation planning and management [97], [98].

4) Social Network Data: With the massive use of social networks, people are generating their digital trace which provides plentiful features of their behavior, social tier, and finally urban dynamics. People are now sharing their spacetime information with others, read and follow the posts of other people in various social network platforms such as Facebook, Twitter, LinkedIn, etc. [46]. In contrast to Facebook, Twitter is a more public profile, and it allows users to dispense their information such as status, modes, news, etc. as a tweet (also with hashtag) which is limited to about 150 characters [23]. Information coming from Twitter can be used for recommendation system, and analysis of sentiment and different social features [21]. 
A wide range of UA can be provided using social network data. The raw form of these data include geo-referenced messages and metadata, and in some cases geo-temporal densitybased information. Geo-tagged information and messages are collected from the social networks using Crawlers. The specific data are collected based on the user's query. These data are stored and arranged in the database based on keywords (e.g., with Hashtags). These data have been applied to identify crowd events [76], [77], [78], [79], [91], transportation planning and management [21], and crowd sentiment analysis [21], [12], [23].

5) Video Data: In the last decades, as a result of increased urbanization and movement of people, large gatherings of people are now observed in different places. Video cameras are installed in the various important places (such as train stations, bus stations, city surveillance, critical infrastructure, etc.) for surveillance.

Therefore, video is an important data source for crowd analysis. Used mainly for crowd tracking and crowd behavior analysis [18], [20], [58], [59], [60], the video data has also been employed in, understanding traffic flow [81], [82], and crowd density estimation and crowd event detection [35].

6) Satellite and UAV Images: The satellite and aerial images are captured by sensors on satellites and cameras on UAV, respectively. The satellites take images at specific times and dates using sensors which pick up photons when they fly over an area. The images can be combined into a mosaic image or captured over multiple days. Examples of imaging satellites include WorldView, Ikonos, Spot, Landsat, etc. The satellite footprint covers a large area on Earth at high resolution.

On the other hand, UAVs can record the real-time activities of ground area from an altitude by taking geo-tagged images and videos. The spatial resolution depends on the altitude of UAV (e.g., high attitude gives a low spatial resolution).

The Satellite and UAV images can provide crowd information over a large outdoor area as they cover a broad geographic region. However, the precision of the crowd information depends on environmental factors such as clear sky index, ambient luminescence, shadowing, etc. These images are employed to automatically analyze crowd count [88], [99], crowd density [89], visual surveillance [100], and urban traffic analysis [101].

7) Other Hybrid Data Sources: Hybrid data sources, as combinations of some of the data sources mentioned above, have also been reported in the literature in forms of- Application on Smartphone for crowd monitoring [90], textitMobile and Twitter data for crowd estimation and urban event detection [22], CDR and Flickr data for attractiveness evaluation of urban tourist sites (e.g., Waterfalls around the New York City) [70], Twitter and Instagram data for detecting ongoing events [91], and Bluetooth and GPS location data for tracking crowd activity and estimating crowd size [92].

Table IV summarizes the various advantages and disadvantages of using different crowd data coming from the sources mentioned above.

\section{StATE-OF-THE-ART OF CROWD DATA ANALYSIS}

This section reviews the state-of-the-art techniques from the literature on the analysis of crowd data generated by the different urban sensors described in section II.

\section{A. Mobile Phone Network Data Analysis}

CDR data serves as one of the major sources of crowd analysis for UED. A subset of CDR record can be taken to analyze and identify crowd events in specific geo-locations. Given that human mostly follow usual routine activities, thus, the intention is to find crowd dynamics from routine trajectories. CDR data only records when a user's activity is detected, and the geo-location of the UEq is pinpointed using the BSs latitude and longitude information. Thus the raw CDR data has space and time sparseness. Different techniques have been reported in the literature to identify user location and trajectory accurately which eventually reduces the space-time sparsity of the data [5], [49], [70].

To identify user's location, Dong et al. defined that each user belongs to a cylindrical cluster with a certain existence probability [5]. Gonzalez et al. considered the users to be concentrated at the centroid of the cell area [32]. Girardin et al. covered all locations by multiple BSs and reported that users were located in the best serving cell, covered by a BS, on a cell map [70]. The measurement has been based on propagation models and cell sectoring factors. Traag et al. also assumed a persistence probability of a user being covered by a specific BS based on a propagation model [49]. In addition, to identify user trajectory, a key issue was to discover a user's pass nodes and most stay nodes (e.g., see Figure 5, office and home location nodes- ' 1 ' and ' 7 ' along with the different pass nodes- ' 2 ' to '6').

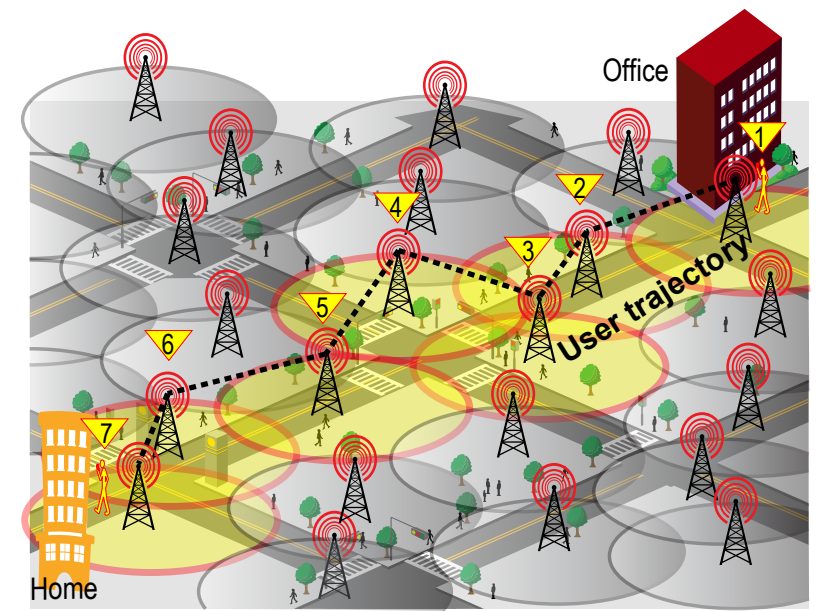

Fig. 5. User trajectory detection using cell tower. ' 1 ' denotes the starting BS i.e., the origin point of the user (e.g., Office), and the destination point is ' 7 ' (e.g., Home). '2' to ' 6 ' are the pass nodes.

The CDR data can be processed in two ways to find the crowd dynamics- a) individual data processing, and $b$ ) group data processing. 
TABLE IV

Pros and Cons of the Comparative Data Sensor for Urban Event Detection

\begin{tabular}{|c|c|c|}
\hline Data Source & Pros. & Cons. \\
\hline \multirow{3}{*}{ CDR } & Estimate huge crowd with high accuracy & Data are network driven \\
\hline & Find the user trajectory & Privacy is major concern as human activity with mobile is highest \\
\hline & Huge real-time data is generated & location accuracy is low compare to RF counterpart \\
\hline \multirow{2}{*}{ GPS } & The system is self-calibrating & GPS suffers from inherent noise \\
\hline & User trajectory information can be recorded & ndoor signal is poor \\
\hline \multirow{2}{*}{ Bluetooth } & Counting speed is higher than RFID & Limited distance (up to $10 \mathrm{~m}$ ) \\
\hline & Better accuracy then RFID & Interference with other RF devices and limited crowd count \\
\hline \multirow[b]{2}{*}{$\mathrm{WiFi}$} & Location coverage is higher than RFID, Bluetooth & Limited crowd can be counted \\
\hline & Estimate crowd size accurately & Interference due to share bandwidth \\
\hline \multirow{2}{*}{ RFID } & Track crowd trajectory & Additional infrastructure is required \\
\hline & Estimate crowd size accurately & No RFID standard, low reading rate \\
\hline \multirow{2}{*}{ Transport } & Estimate crowd count with high accuracy & Data are network driven \\
\hline & & Private transports passenger information are missing \\
\hline \multirow{3}{*}{ SN } & Sentiment analysis & Different language and informal words are difficult to analyze \\
\hline & Find social-tier & Crowd estimation is not accurate \\
\hline & Data are user driven & location accuracy is low compare to RF counterpart \\
\hline \multirow{2}{*}{ Video } & Analyze crowd dynamics accurately & Placement of the camera ambient lighting are important \\
\hline & Estimate crowd size accurately & Accuracy depends on image and separation \\
\hline \multirow{2}{*}{ Satellite } & Estimate huge crowd with high accuracy & Indoor crowd size cant be approximated \\
\hline & & Data transmission interval is about 6 hours \\
\hline \multirow{2}{*}{ UAV } & Cover higher outdoor area & Limited flight time and no indoor coverage \\
\hline & Estimate real-time outdoor crowd accuracy & Interrupted by environmental conditions \\
\hline Census & Very accurate spatial resolution & Dated-out data as data are collected after 10 years interval \\
\hline
\end{tabular}

a) Individual data processing: In this technique, most frequently visited places or most stay locations (e.g., office and home), pass locations and thereby the daily mobility pattern of each inhabitant can be found from CDR raw data. Two features identify Home and Office, one is the frequency of user contacting with a BS during the evening and daytime respectively, and the other is the duration of stay at a place. Some techniques have also been proposed in the literature to find these places [29], [70], [96], [71]. This information can be validated using the census data. By connecting several pass nodes and stay nodes, the individual trajectory can be constructed. Gonzalez et al. reported spatial and temporal regularity in daily human mobility which can be represented using simple regular patterns [32]. The same principle was used to estimate the daily mobility tier which can be used to find the O-D matrices for the transportation [71]. Schneider et al. investigated a method by combining the daily mobility patterns, called motifs, of different large-scale data sources which can be extended to a range of UA [53]. In each dataset, the authors observed ubiquitous daily mobility patterns which were reproduced statistically with an analytical model. Zhao et al. proposed a supervised statistical learning based framework that identified individuals' trips from CDR data [25]. Toole et al. [64] and De Mudler et al. [102] proposed techniques which extracted user movement profiles with high accuracy from the CDR data. Also, there exists tools to identify and validate road usage patterns from O-D matrices [64].

b) Group data processing: By combining some cell tower information, events can be detected. Elzen et al. proposed a tool to identify clusters of cell towers with identical call patterns to detect events [66]. Xavier et al. related human mobility and workload dynamics from CDR data in case of large events for better management of UA [72].

Isaacman et al, [65] explored the CDR data to find the frequency of travel back and forth between Los Angeles and New York. They have identified how people travel in and around these cities which were helpful for the urban and transport planning. Louail et al. [67] and Isaacman et al. [68] utilized the CDR data to pin down in the city's hotspot, spatial structure and the busiest points during particular hours of a day. Di Lorenzo et al. optimized a transit network based on mobility patterns of people extracted from CDR data which can help government agencies to manage the transport network efficiently [69].

\section{B. RF Data}

RF data has been an important source for UA like- crowd mobility tracking, and accurate crowd size count. However, the RF data generation is 'user participatory', therefore, the accuracy depends on the participation of the individuals in the crowd. Schematic diagram of data generation and processing is shown in Figure 6.

a) WiFi and Bluetooth: WiFi access point (AP) can monitor a crowd with WiFi activated handheld devices. The footprint of the WiFi can include a certain number of people. With the significant number of WiFi grid, the user-driven crowd size can be estimated precisely [11], [13]. Weppner and Lukowicz [6], Eagle and Pentland [7] and Weppner et al. [11] presented frameworks to estimate crowd density based on users equipped with Bluetooth enabled handheld devices. Bluetooth can be used to assess crowd density with an accuracy of about $75 \%$. Versichele et al. [9] employed Bluetooth scan to analyze the crowd trajectory in a mass gathering. This can be used to create visitor flow maps from several mutually exclusive visitor's profiles. User's participatory Bluetooth hardware can be used to count passenger and record O-D matrix of travelers' journey. These data can be used to optimize transport planning [73]. 

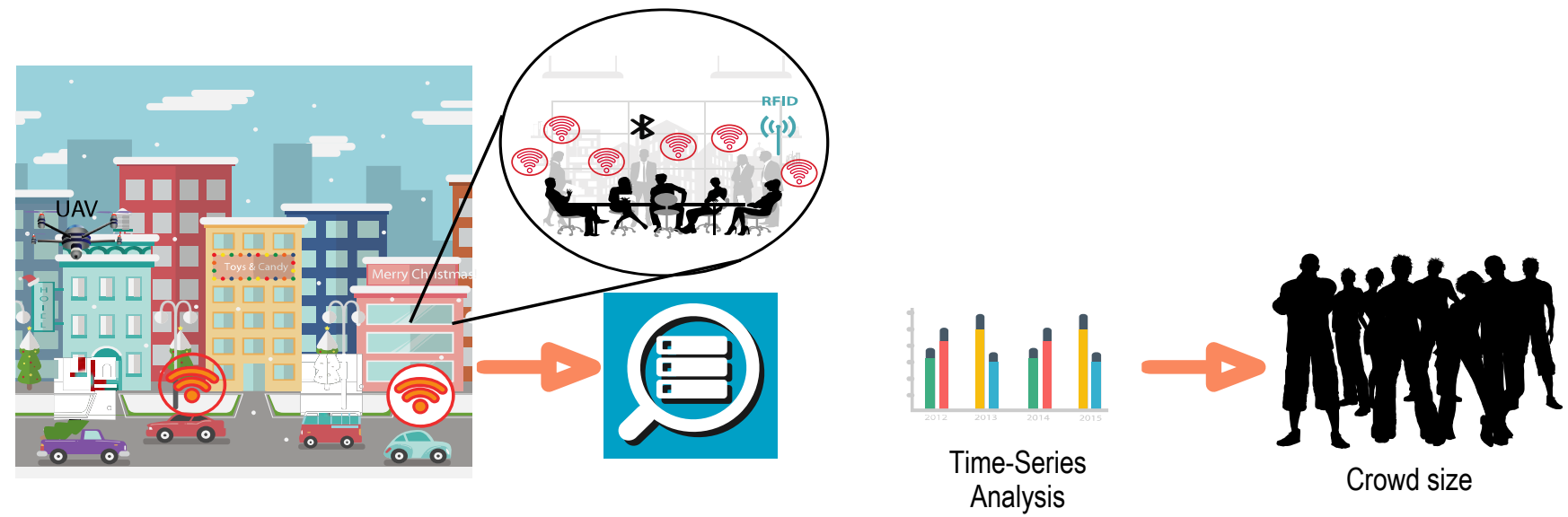

(a) Cartoon of the RF data generation process

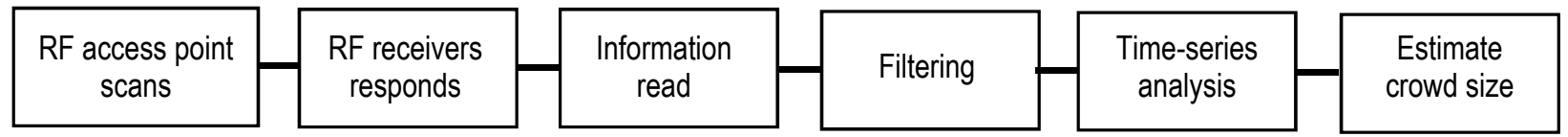

(b) Block diagramatic representation of the RF data generation and processing steps

Fig. 6. RF data generation and processing technique. The crowd size estimation steps using different RF sensors in a room are shown in (a) and the block diagrammatic representation of each step of RF data generation and processing technique are illustrated in (b). In many of the RF system, additional RF infrastructure is required. If all the user-participated, it can estimate a crowd size accurately.

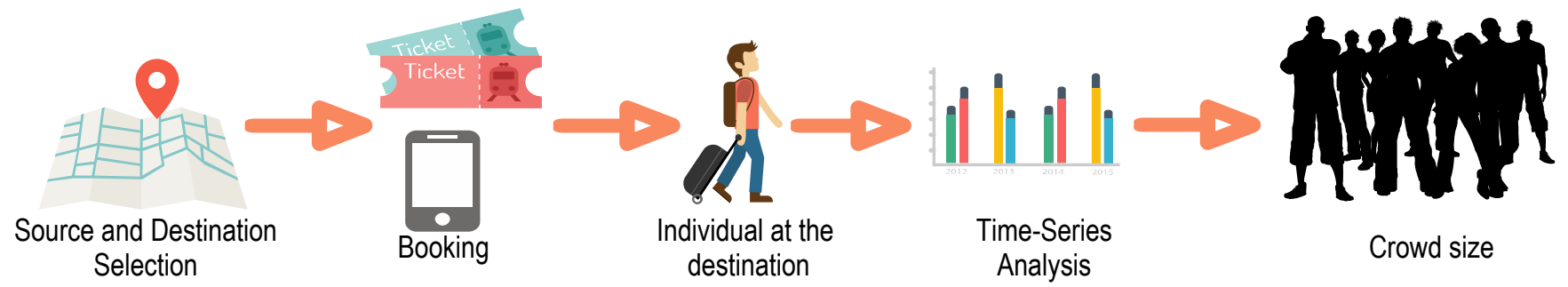

(a) Cartoon of the transportation data generation process

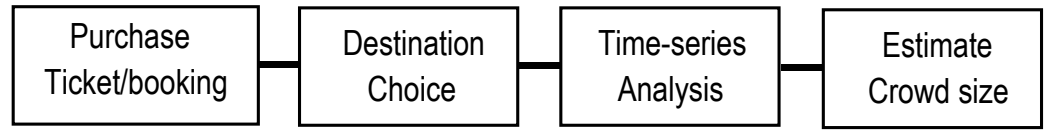

(b) Block diagramatic representation of the transportation data generation and process

Fig. 7. Transport data are generated when a ticket is purchased and booked. By analyzing these data for a particular destination, a crowd event can be detected.

b) GPS: The GPS data can be utilized to perceive the pedestrian motion and estimate outdoor crowd sizes [14], [90]. A Geographic Information System (GIS) is integrated with GPS to illustrate the crowd visually [14].

c) RFID: Crowd dynamics including counting and tracking are also possible using RFID data. Mowasafi et al. introduced a framework to detect both static and dynamic crowds using RFID and found some significant crowd properties [50]. The human crowd density estimation algorithm was then trained with mass crowd event data. The accuracy of the algorithm was determined to be above $70 \%$. Michau et al. outlined a procedure to extricate vehicular trajectories and trip information, and they have recuperated the vehicular trajectories with the accuracy of $84 \%$ [10]. d) $O W C$ : The OWC can also be used for crowd counting Yang et al. introduced a nonparametric statistical method to detect pedestrian automatically with high accuracy [17]. The method has improve the accuracy of the counting.

\section{Transport Data}

The transportation data can be treated as time-series data, collected from transportation and parking lot databases, about the number of people coming to a destination. Therefore, time series analysis methods can be largely applied on transportation data for UED (see Figure 7 for generation and processing steps).

In [74], the author used London Overground network data to manage and plan a contemporary urban railway service and 


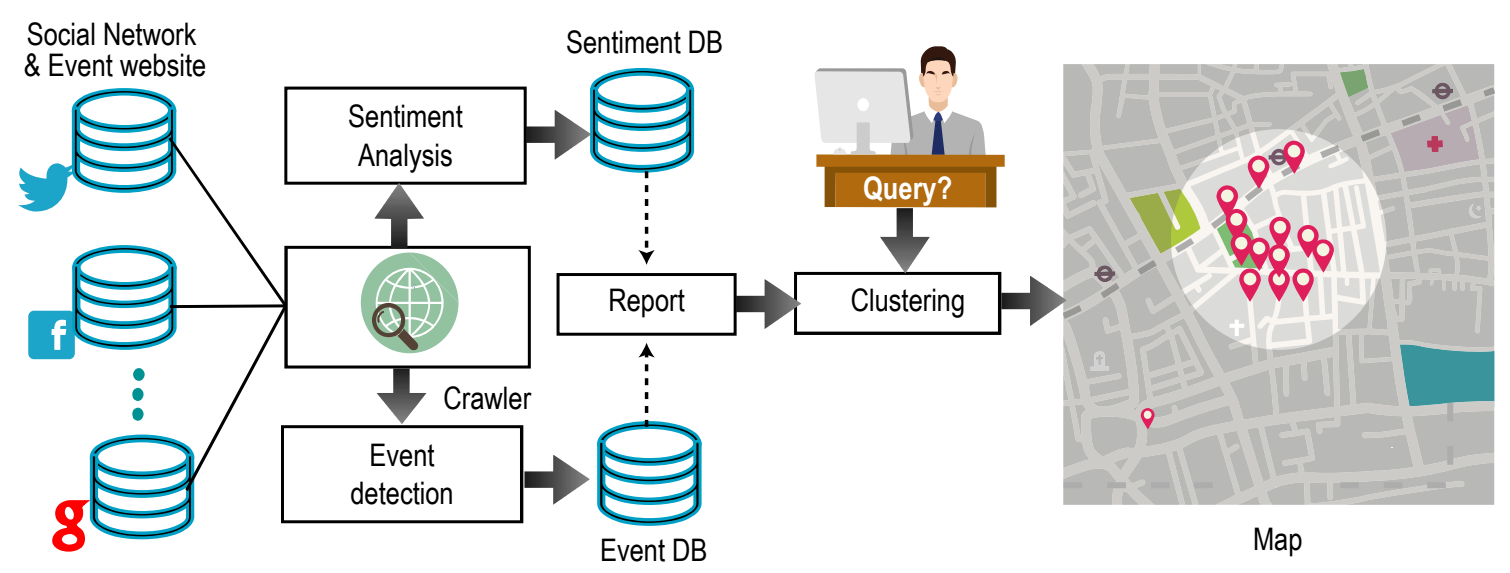

Fig. 8. Block diagram of geo-temporal observation of social network messages. The crawler periodically retrieves a set of event related information and the user's sentiment about these events from different social network platforms based on the queries. The retrieved spatio-temporal geo-tagged event information is stored as a tuple field in the sentiment and event database. The textual field contains the semantics of message with hashtags. The collection of messages that satisfy the user's queries are stored as reports. A suitable clustering algorithm clusters the report at the end.

showed its potential impact. A framework was introduced for the timing of trip based on sets of analyses and transportation service plan. This work was also used to estimate the crowd density and the crowd event. Fare card data have been used to understand the mobility patterns of commuters. Nassir et al. proposed a heuristic algorithm to find the O-D matrix and estimated the actual travelers mobility. The algorithm was helpful for better comprehension of the mobility motifs and path options of the travelers [2]. Pereira et al. also proposed a framework to detect crowds using public transport smart card data by comparing habitual and non-habitual behavior of the crowds and to assist the transport manager in mitigating disruptions due to crowd-hotspot [51]. The framework periodically retrieved possible explanatory reasons for the crowdhotspot using text analysis of social networks and event website text data. Finally, a probabilistic model is used to estimate over-crowding. Public bicycle system data was used to find the individual's movement and the results were illustrated using a color legend. This approach identified the individual's mobility pattern and estimated the most visited destinations [75]. Tirachini et al. examined different aspects of passenger crowding in transport management and operation [52]. They considered waiting and in-vehicle time saving, route and bus choice, travel time reliability, passengers' wellbeing, vehicle size, frequency, and fare. The effect of crowding was found to be related to demand and vehicle time-saving prediction. The authors estimated the passenger's discomfort on waiting time at the stands, riding time to the services, travel time and fare, and impact of crowding phenomenon on the health of passengers. All these can be used to estimate the number of the passenger for an O-D matrix and a mobility pattern.

\section{Social Network Data}

From the social network data collected using Crawlers allow a user to perform sentiment analysis and event detection. Figure 8 shows the conceptual flow diagram of Social Network Data analysis for UED.
The sentiment analysis employs text analytics, natural language processing, as well as computational linguistics to extract subjective matter from the text to be analyzed. In this case, Hashtag messages (or tweets) shared by users related to an event or an opinion about a service are collected and stored in a sentiment database. The sentiment of people are evaluated via different sequential steps: firstly, collected messages are preprocessed and then unnecessary words, preposition and punctuations are removed. Secondly, preprocessed messages are transformed to a feature vector. Thirdly, these features are analyzed through Machine Learning algorithms. Finally, the classified output is stored in the sentiment database [21].

A significant number of research articles have been found on crowd analysis based on social network data. Arcaini et al. suggested geo-temporal density-based technique to identify information of regular and irregular incident announced on Twitter [76]. The suggested method analyzed user generated geo-referenced messages about these (a-)periodic events (i.e., user participatory) as well as other geo-referenced metadata. Finally, the (a-)periodic crowd events are identified. The work done by Candelieri and Archetti considered the streams of Tweets on urban transport operations [21]. The proposed framework can accumulate Tweets and analyze them to detect crowd events or to understand the sentiment of the people. Authors have tested their framework for an urban transportation in Milan. The sentiment inferred by the passengers can help the transport providers to modify their mobility planning [21]. Alp and Oguducu introduced an algorithm to analyze Tweets for UED [23].

$\mathrm{Xu}$ et al. proposed a Bigdata model, characterized by $5 \mathrm{Ws}$ (What, Where, When, Who, and Why), to detect urban events at real-time at a geo-location from social network data [77]. The spatiotemporal information are extracted and used for a UED, then the event is presented by GIS-based annotation. The model has been verified by real-life data. With the increased sharing of location and opinion of citizens on the social network, the Location-based Social Networks (LBSN) has gained interest by the urban planners to plan upcoming events. 
Khalifa et al. combined density-based clustering, social data mining, and outlier detection to detect crowds in cities at realtime [78]. This method has been validated by Twitter data of New York City on a reference (e.g., any) day and on study day (e.g., New Years Eve) when crowd events are expected.

A mention-anomaly-based event detection (MABED) method was proposed to detect events from Twitter data. In this method, the period of each event was estimated from Twitter. Authors reported linear run-time for the model in corpus size, and improved readability of the event description through highlighting main words. The model helped to understand the interest of users and designed three visualizations to track the detected events [79].

In short, the geo-tagged information collected from the social networks are clustered and mapped using GIS.

\section{E. Video Data}

Stationary crowd-group analysis to estimate the stationary time of foreground pixels was proposed to perceive crowdscene and the effect of stationary groups on traffic flow [81]. The accuracy of the method depends on camera position, image processing, and dynamic nature of the crowd group.

Loy et al. [80] studied as well as compared the state-of-theart regression methods dealing with many co-linearity among features. They found that feature selections are important and depend on crowd scene. These selected features can be used for image-based crowd counting at stations. The accuracy depends upon camera orientation and ambient light condition.

The survey by [59] gives an investigation of physics and state-of-the-art bio-inspired techniques of crowd mobility pattern. Authors have reviewed crowd density estimation and mobility pattern analysis methods and provided some research directions. A survey on pedestrian movement (i.e., flow) has been proposed for crowd dynamics model [103], [104]. The model is used to design public traffic systems for effective crowd dispersal. Authors have reviewed pedestrian movement and crowd mobility pattern. The article in [35] surveys on crowd analysis using computer vision and shows crowd density estimation, crowd event detection and validation techniques.

A deep learning based approach combining deep and shallow fully convolutional network was used to extract high and low level features in an image. The high low and level features included face/body and blob, respectively. Finally, the crowd size was estimated from a dense crowd image [82]. Figure 9 shows the taxonomy for crowd analysis methods. There are mainly two types of approaches reported in the literature for crowd size estimation [35], [37]: direct detection or object detection based approach, and indirect or feature based approach (Figure 9).

In the direct method (see Figure 10 (a)), the main steps for the detection are: segmentation, detect the individuals present in the scenes, and count the people using classifiers (see Figure 9 (a)) [105], [106], [107]. The model-based and trajectory clustering based approaches belong to the direct method. In the model-based analysis, a model is used to segment and detect and then count each individual [37], whereas, in the trajectory

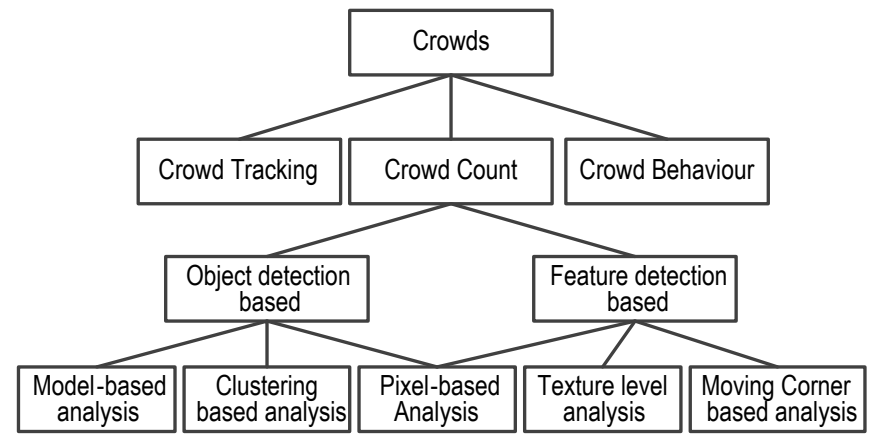

Fig. 9. A taxonomy for crowd analysis methods. Different direct and feature based crowd counting methods are illustrated.

clustering based approach, each independent motions in the image are detected using clustering the site of interest, and finally count them [54].

In the indirect method, the main step is to extract several features (local/holistic) from the images (Figure 10 (b)). Pixelbased, texture-based, and corner-point-based analyses belong to the indirect method. Pixel-based analysis deals with local features to approximate crowd size in a crowd image. However, most of the researchers pay attention to approximate the crowd-density rather by removing the background in the first step or generating an automatic background. For indoor video images, authors in [83] and [84] estimated crowd size employing a linear regression model and a feed-forward neural network method respectively. Both papers have considered foreground pixels as well as edge. For outdoor video images, authors in [85] employed a linear regression model and foreground pixel as an image feature, and in [86] employed a back propagation neural network and foreground segmentation as well as edge detection as an image feature. In texturebased analysis, texture is considered as an image feature. This method explored a coarser grain and required image patches analysis. Texture-based crowd estimation systems for both indoor and outdoor applications are given in tabular form in [37]. In corner point based analysis, the researchers utilized interest points (as feature) for crowd detection, and these features are masked with optical flow model [87].

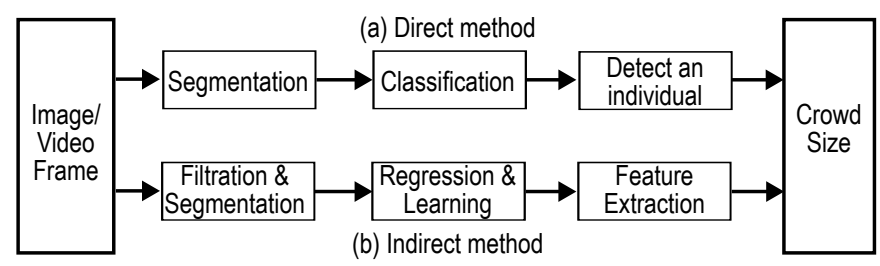

Fig. 10. Block diagram of (a) direct and (b) indirect method of crowd analysis from video frames or an image. In the indirect method, features are extracted from the image which estimates large crowd size accurately compared to the direct method.

\section{F. Satellite and UAV Image}

The following subsections discuss processing and analysis of Satellite and UAV Images to estimate crowd event and crowd size. 


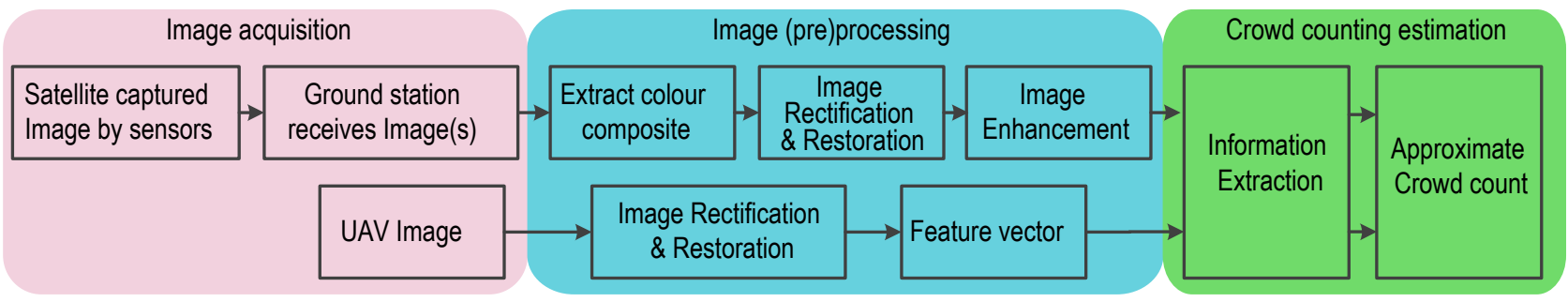

Fig. 11. Block diagram of Data generation from Satellite and UAV and processing steps.
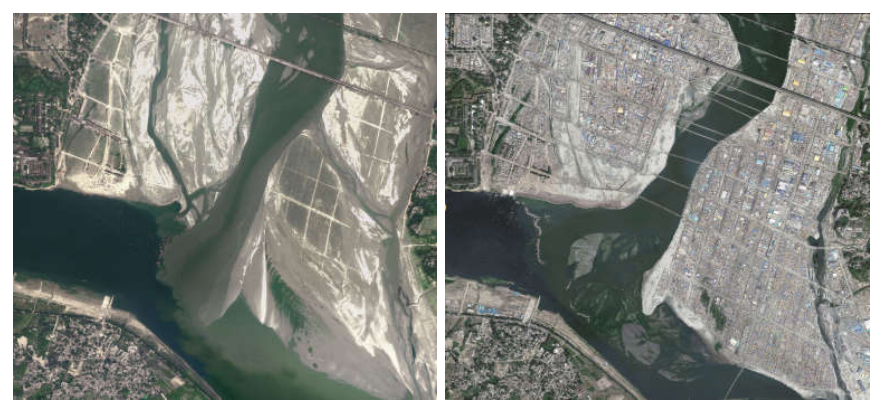

Fig. 12. The Kumbh Mela in Allahabad (left) on October 2012 before the mela and (right) on February 2013 during the mela (source: http://s.ngm.com/ 2014/02/kumbh-mela/index-FINAL.html).
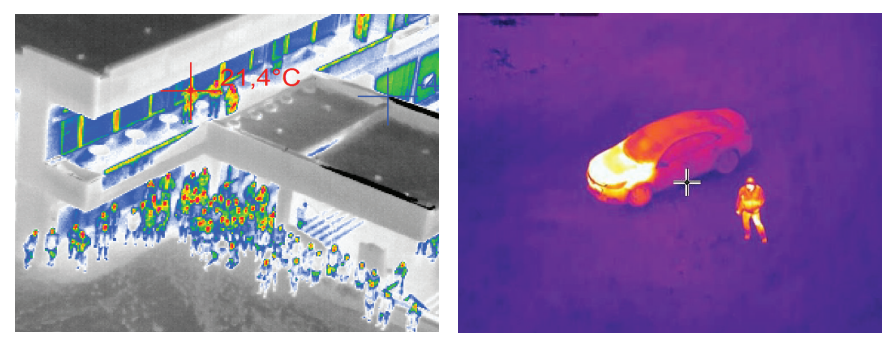

Fig. 13. Example of two UAV images captured by thermal cameras. Crowd size at $21.4^{\circ} \mathrm{C}$ (left image, source: https://www.drone-thermal-camera. $\mathrm{com} /$ ) and moving vehicle at $12.5^{\circ} \mathrm{C}$ (right image, source: https://www. drone-thermal-camera.com/).

1) Satellite: Satellite image processing is time-consuming due to their very high resolution, and successive images are difficult to obtain due to processing time and atmospheric conditions (see section II-B6). The images captured by a satellite is sent to a ground station where image data is stored in Raster format. The color composites are extracted from the image which has been pre-processed for geometric and radiometric connections, then digital image processing techniques have been employed to enhance the quality of the image. Finally, information are extracted from the image (see Figure 11).

Figure 12 shows exemplary satellite images of Kumbh Mela, which was held at Allahabad, India during February 2013. The area before the mela in October 2012 (left) and during the mela in February 2013 (right) are seen in the images. The right image can be used to estimate the crowd size and crowd event.

The crowd event and crowd size can be detected from
Satellite Images with a probabilistic framework. Sirmacek and Reinartz proposed a novel approach to detect crowd automatically from very high-resolution satellite images by using airborne sensors [88]. In this method, the local features were extracted from intensity and color bands of the image. Authors used three different information (e.g., digital elevation model or DEM, street segments, and shadow) for feature selection through which they could remove redundant local features. Then a probability density function (pdf) using Gaussian kernel functions with adaptive bandwidth selection method was suggested. The proposed robust algorithm can deal with input images of different resolutions and can detect crowd automatically using pdf function based thresholds. Worldview2 satellite images taken over Cairo and Munich cities were used for testing this method, and the authors reported a very high accuracy.

2) UAV: The UAV images are recorded with an image coordinate (latitude, longitude) information which requires to be converted to a standard coordinate such as Universal Transverse Mercator (UTM) coordinate system. After the geometric correction, the crowd size is estimated from an image or a frame in the video using similar method discussed in section III-E.

Figure 13 shows two thermal images taken by UAV cameras.

Meynberg et al. [89] proposed texture classification methods to identify crowded regions from aerial images. As it is hard to get the correct size of an individual from such an image, the authors proposed multi-class texture classification using four classes or groups of defined crowd density ranges. Authors reported an accuracy of $97 \%$ using both patch-based Bag-ofWords features, and an SVM with filter-based Gabor features.

\section{G. Information Fusion and Hybrid Data}

In UED, hybrid data plays an important role and information fusion of data coming from hybrid data sources improves the estimation accuracy of the crowd size and UED. For example, web-linked fixed and mobile sensors accumulate different information from the urban space, and the information is key for detecting urban events at various geo-locations. Using different types of fusion techniques, information acquired from various urban sensors can be fused based on some fuse rules to get better, complete, appropriate and accurate information. Moreover, this fusion technique reduces the uncertainty of the decision support system. 
1) Information Fusion: Information fusion is a technique of integrating information extracted from heterogeneous sources (e.g., the ones described above, see sec. II-B) with different contextual and conceptual representations to generate a new and enriched information set by overcoming the information deficiencies of individual datasets. There are three types of fusion methods [108], [109]: a) complementary fusion, b) redundant (also called competitive) fusion, and c) cooperative fusion.

a) Complementary Fusion: When two or more individual sensors (e.g., $S r_{1}$ and $S r_{2}$ ) extract complementary information (e.g., $D_{1}$ and $D_{2}$ ), and the information are merged to acquire a more complete or broader information (e.g., $D S$ ) of the environment (Figure 14 (a)), such fusion is called complementary fusion. For example, in a video surveillance system operating at a station, videos are collected (e.g., $D_{1}$ and $D_{2}$ ) from multiple independent cameras (e.g., $S r_{1}$ and $S r_{2}$ ) and fusing them provides the complete picture of the station (e.g., $D S$ ) (Figure 14 (a)).

b) Redundant Fusion: Redundant fusion, also called competitive fusion, aggregates similar information collected from same urban space by independent sensors (e.g., $S r_{1}$ and $S r_{2}$ ) to enhance the accuracy of the information (e.g., $D S$ ) (Figure 14 (b)). In WSN, redundant fusion technique is employed to enhance the accuracy and reliability of information as well as to improve energy saving by preventing transmission of redundant information [108].

c) Cooperative Fusion: The cooperative fusion method collects different information from same urban space using multiple separate sensors (e.g., $S r_{1}$ and $S r_{2}$ ) that would not be obtainable from the individual sensors (e.g., $D_{1}$ or $D_{2}$ ) (Figure 14 (c)). In collaborative communication, the quality of service of the wireless network is greatly improved by this fusion method [110]. Moreover, this is also used for identifying the position of a wireless receiver (by the APs) on the urban space accurately using triangulation principle.

2) Hybrid Data: Hybrid data can play an important role in crowd analysis, provided, they are fused appropriately based on their sources. The following paragraphs illustrate the usability of fused hybrid data in crowd analysis.

a) Data from GPS and App on Smartphone: Wirz et al. [90] introduced a location-aware crowd monitoring approach using smartphones with an app installed on it during mass gatherings which can be a replacement for video-based solutions. A participatory approach has been considered where all individuals share their location information on a voluntary basis. The only limitation is that all individuals may not share their location information. Thus authors used fused data from the app and GPS trajectory to monitor the crowd. They have used pedestrians' speed (from the GPS data) to estimate the crowd count as well as the crowd density. This method can assess the actual crowd size.

b) Mobile and Twitter Data: Botta et al. [22] used CDR/IPDR and Twitter data to estimate crowd in an urban event. Their findings depicted that the accurate number of attendees may be extrapolated in a geo-location from CDR/IPDR and/or Twitter data.

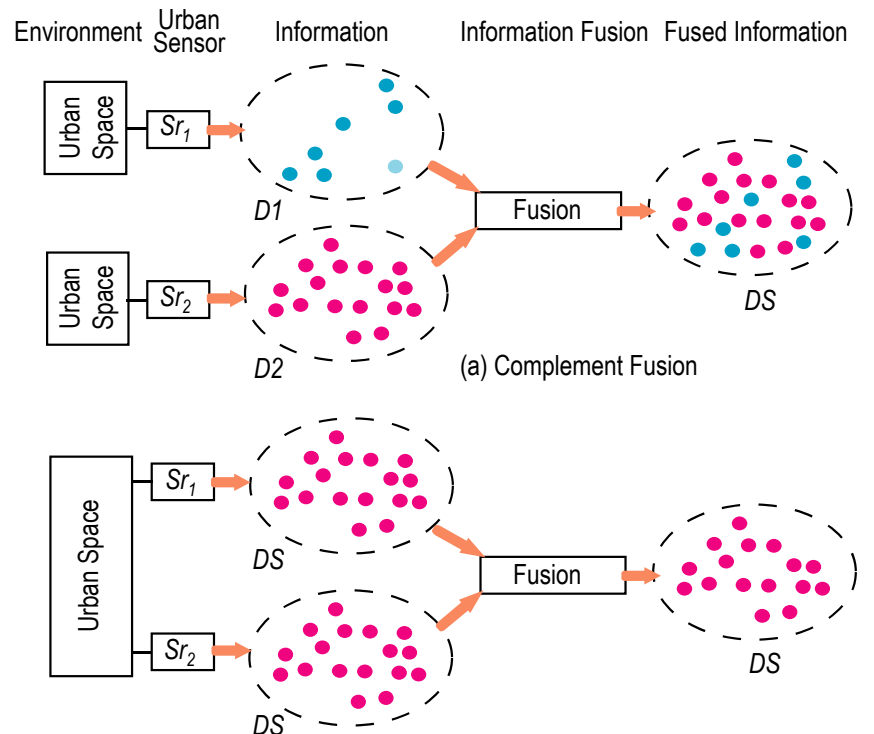

(b) Redundent (or Competitive) Fusion

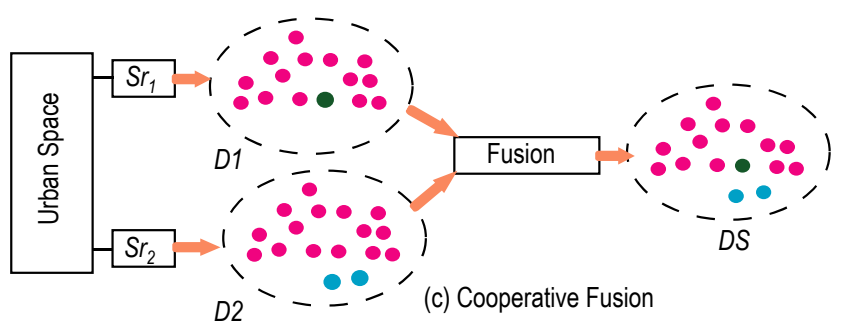

Fig. 14. Different types of Information Fusion techniques: (a) Complementary fusion, where complete information is achieved by fusing information of multiple independent sensors; (b) Redundant fusion, where the accuracy as well as the reliability of the information are improved by fusing same information of independent sensors; and (c) Cooperative fusion, where better and accurate view of the same space information is collected using multiple separate sensors.

c) CDR and Flickr Data: Girardin et al. [70] has employed a method to use the CDR data of AT\&T and photos posted on Flickr to find the urban attractiveness around the New York City Waterfalls.

d) Twitter and Instagram Data: Ranneries et al. [91] presented a novel approach with increased classification accuracy for UED which fused Twitter and Instagram data and detected an ongoing event based on posts submitted during the last one hour.

e) Bluetooth and GPS Data: In yet another approach, data from Bluetooth scans and GPS locations of handheld devices are fused and analyzed to track crowd activities and estimate the crowd size [92]. The authors also introduced a novel crowd visualization tool.

\section{OPEN ACCESS DATASETS AND APIS}

Crowd analysis and management are essential for planning UAs pertaining to a smart city. In these applications, APIs play a key role as they are the doors to interoperability across different organizations to connect, share, and provide real-time data handling capabilities. In this section, some of the popular open access benchmark public datasets (see Table $\mathrm{V}$ for a 
summary) and few major APIs (see Table VI for a summary) are listed below which have gained considerable importance for the UED and DSS design for different UA.

TABLE V

Benchmark Data Sources, Datasets, And Their Applications

\begin{tabular}{|c|c|c|c|}
\hline Source & Dataset & Applications & Ref. \\
\hline \multirow{2}{*}{ CDR } & D4D Challenge on Mobilephone & Cr. Co. \& Trj. & [111] \\
\hline & Nodobo Data & Soc. Gr. & [112] \\
\hline \multirow{6}{*}{ Video } & Data-driven Crowd Analysis & Cr. Trj. & [18] \\
\hline & Extremely Dense Crowd Images & Cr. Co. & [113] \\
\hline & QUT Crowd Counting & Cr. Co. & [114] \\
\hline & US Highway 101 & Veh. Trj. Con. & [115] \\
\hline & CUHK Crowd & Cr. Sz. & [116] \\
\hline & EyeCrowd & Cr. Co. & [117] \\
\hline \multirow{3}{*}{ Transp. } & US Government's Open Traffic $^{1}$ & Traf. Fl. Co. & [118] \\
\hline & Datagov Ireland $^{1}$ & Trf. Co. & [119] \\
\hline & London DataStore $^{2}$ & Trf. Co. Con. & [120] \\
\hline UAV & UAV 50 and UAV123 & Cr. Trk. & [121] \\
\hline \multirow{2}{*}{ Hybrid } & CDR, Tweets, News & Cr. Co. Trj. Sen. & [122] \\
\hline & CDR and Tweet & Cr. Sz. \& UED & [123] \\
\hline Census & Cambridge Open Data & Cr. Co. & [124] \\
\hline Survey & TravelTracker & Trn. Pln. & [125] \\
\hline
\end{tabular}

1 in the form of Spot count; 2 in the form of O-D matrix; Legend- $\mathrm{Cr}$. Co. Crowd count; Cr. Trj.: Crowd trajectory; Soc. Gr.: Social Graph; Sen:

Sentiment; Vh. Trj. Con.: Vehicles trajectory and congestion; Cr. Sz.: Crowd size; Trf. Fl. Co.: Traffic Flow Counts; Trf. Co.: Traffic count; Ev. Det.: Event detection; Est. Trf.: Estimate traffic; Trn. Pln.: Transport planning.

\section{A. Benchmark Datasets}

a) D4D Challenge on Mobile phone [111]: The dataset contains five months (from 1 December 2011 to 18 April 2012) CDR data from five million of Orange's customers in Ivory Coast. It gives details of hourly antenna-to-antenna traffic, the trajectory of fifty thousand users for two weeks and 500 thousand customer's trajectories for the whole observation period. This dataset can be utilized for crowd counting.

b) Nodobo Data [112]: This dataset incorporates mobile phone usage of 27 students from September 2010 to February 2011, and contains 5292103 presence records, 13035 call records, 83542 message records and other data. This will help to find the social graph.

c) Data-driven Crowd Analysis [18]: This dataset contains crowd videos downloaded (or crawled) by search engines as well as archive footage websites. This includes 100 individuals' trajectories selected randomly from all moving people.

d) Extremely Dense Crowd Image [113]: The dataset incorporates 50 still images collected from the public web and Flickr with head counts per image ranges from 94 to 4543 with average head counts of 1280 per image.

e) QUT Crowd Counting [114]: This annotated dataset contains videos taken by three cameras placed at different ambient conditions and different viewing angle at the QUT campus. This QUT datasets can be used to train a model which estimates crowd size in hard ambient condition.

f) US Highway 101 [115]: The US Highway 101 Dataset is a part of the Next Generation SIMulation (NGSIM) program and contains very high-quality traffic data. This includes three datasets of vehicle's trajectory and congestion data, data about the interactions of travelers and other information of the surroundings. g) CUHK Crowd [116]: The Chinese University of Hong Kong crowd dataset contains 474 crowd video clips from 215 scenes with varying densities and perspectives. The videos have been taken at many different environments, such as, roads, malls, public parks, and airports.

h) EyeCrowd [117]: The dataset contains 500 indoor and outdoor images with different crowd sizes and each image may contain up to 268 faces. It also provides rectangular faces labels, and eyetracking data.

i) US Governments Open Traffic [118]: This site gives a wide range of traffic data which includes transportation, traffic, highways, local roads, etc. at the Federal, County, and City levels. Also available weekday daily traffic count information of some of the major U.S. cities based on spot studies. The count is averaged and includes some selected arterial streets.

j) Datagov Ireland [119]: This site includes national road traffic count of 300 roads, transport dataset of Ireland.

k) London DataStore [120]: This sites presents information about some bicycle hires, public transport journey, metro network performance data, and schedule of public transport.

l) UAV 50 and UAV123 [121]: UAV 50 and UAV123 are low altitude areal video datasets and benchmark for low altitude UAV target (such as object/human).

m) CDR, Tweets, and News [122]: This dataset contains data from two Italian cities, i.e., Milan and Trento. It contains the CDR data, from 1-11-2013 to 25-12-2013, of Telecom Italia cellular network for Milan and Trento. This dataset gives information about some interactions between mobile users of Telecom Italia for different area of Milan. It contains mobile users' interaction data from 1-11-2013 to 24-11-2013 and the volume is $138.8 \mathrm{~GB}$, news portal data MilanoToday from $01 / 11 / 2013$ to $31 / 12 / 2013$, tweets data emanated from Milan city during the months of November and December.

n) CDR and Twitter [123]:: This dataset contains mobile phone and Twitter activity data of Milan from 1 November 2013 to 31 December 2013 which can be used to see the relationship between crowd, mobile phone and Twitter.

o) Cambridge Open [124] : It comprises two years (2014 to 2016) monthly parking ticket information; Average Daily Traffic Counts from the year 1972 to 2014; 2016 Land Use Data. These mentioned datasets might be used to estimate a baseline for designing a DSS for UA.

p) Travel Tracker [125]: The CMAP has surveyed voyage related activities for northeastern Illinois. The data has been collected from January 2007 to February 2008 where 10,552 households participated in the survey.

\section{B. APIs Suitable for Crowd Data}

a) Google [133]: Google APIs allow to access different services provided by Google, e.g., Google Maps, Google Earth, etc. The API supports data analytics, machine learning as a service or access to user driven data. Google API can be exploited for web mapping and shows geo-location. In addition, Google Trends [126] provide public Google Search trends which might be helpful in finding out inhabitant sentiments for a detected urban event and used for appropriate UA planning. 
TABLE VI

LIST OF APIS FOR CROWD DATASETS

\begin{tabular}{cclll}
\hline Ref. & Launched & API Name & Method & Usage \\
\hline$[126]$ & 2006 & Google Web Search & Google Search & User Sentiment \\
\hline$[127]$ & 2015 & TFL Unified & O-D matrix & Crowd \& and congestion detection \\
\hline$[128]$ & 2014 & Big Open Data & CDR, News-portal \& tweets & Crowd event detection \\
\hline$[129]$ & - & REST & Read Tweets & User Sentiment and Crowd event \\
\hline$[130]$ & 2004 & Flickr & Image/Video & Estimate crowd size \\
\hline$[131]$ & 2016 & Transport & Travel and Ticketing & Estimate crowd size \\
\hline$[132]$ & 2010 & Bing Maps & - & Web mapping \\
\hline$[133]$ & 2010 & Google Maps & - & Web mapping \\
\hline$[134]$ & 2016 & Crowd REST API & - & Crowd information \\
\hline
\end{tabular}

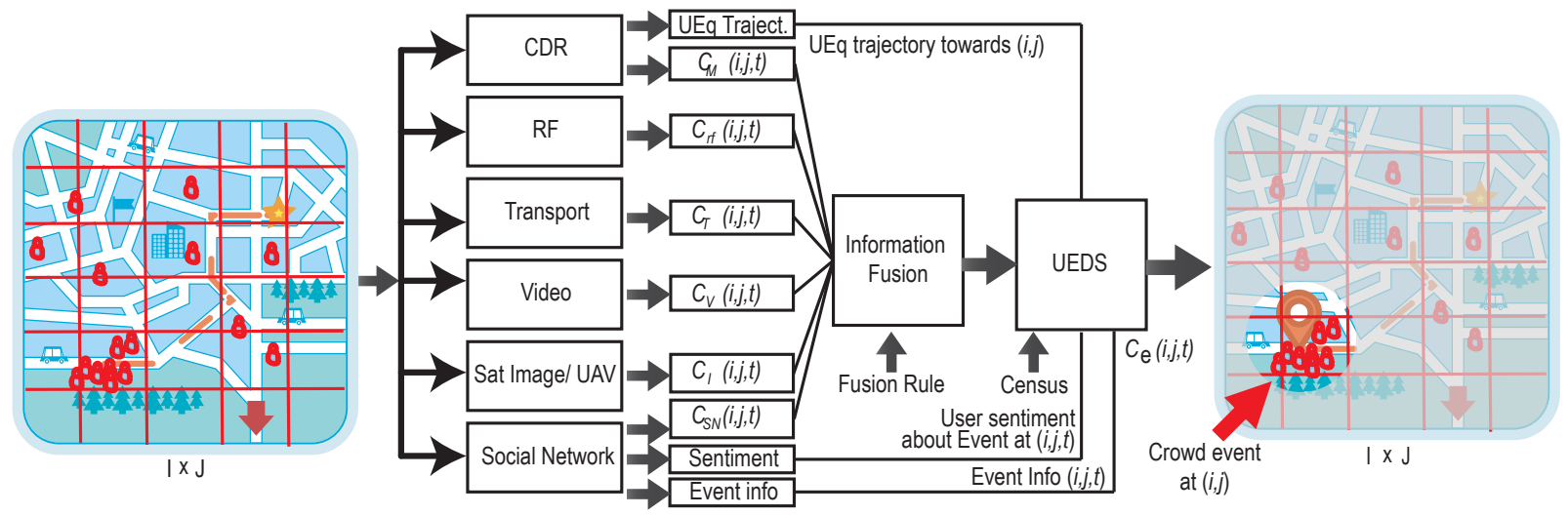

Fig. 15. The UEDS detects an urban event in a geo-location. The UEDS utilizes the fused information of all urban sensor data, census data, users' sentiment about an event, event information published in social network and event webs, and detects an event at a geo-location $(i, j)$ and estimates $C_{e}(i, j, t)$.

b) TFL [127]: Transport For London (TFL) unified API supplies source and destination data of bike hire, different transport modes, and road information.

c) Dandalion [128]: At early 2014, Telecom Italia shared the telecommunication activity, social activity and published news article datasets of Milano and Trento through Dandalion API.

d) REST \& Streaming [129]: The REST APIs give a limited access to reading Twitter data, follower's data, and user's profile. The APIs recognize Twitter applications and users using 'OAuth' and send responses are in 'JSON' format. The streaming APIs provide developers an access to Tweet data with a low latency.

e) Flickr [130]: Flickr is a free web hosting facility suited for images as well as videos. Photo researchers and bloggers widely use this. Through the API, Flicker information can be used to estimate crowd size.

f) Bing [132]: Bing Maps servers are exploited for web mapping and enable to search, explore, share information of the geo-locations. Therefore, this API can serve to interconnect many services based on Bing.

g) Crowd REST [134]: This API allows to develop own REST crowd datasets using Atlassian REST plugin. Finally these data can be integrated into DSS to design different UA. It allows access to crowd data via URL paths. This can be integrated to a REST API and in this case the default format will be XML/JSON.

The summary of the APIs used for crowd analysis and management are listed in Table VI.

\section{Support System For URban APPliCATIONS}

An important part of delivering a UA connected to a crowd event is the SS. Below a generic framework for UEDS is described which is required for designing a DSS for an appropriate UA.

\section{A. Urban Event Detection System}

Consider a geographical area is divided into $I \times J$ small cells. Census data along with daily periodic crowd data indicate that each cell contains $C(i, j, t)$ crowd at time $t$, so this is the baseline for that $(i, j)$ cell area at time $t$. The goal of the UEDS is to estimate crowd size $\left(C_{e}(i, j, t)\right)$ in order to detect an urban event.

However, for accurate estimation of the crowd size, redundancy from the data must be removed. It is possible that one user is involved with multiple activities at a geo-location in the urban space, e.g., a user is using Twitter and calling at the same time. In this case, duplicate data are generated and matched users need to be excluded from the estimation of crowd size. An efficient approach to handle data redundancy in such situations is to apply information fusion on different datasets.

The concept is illustrated in Figure 15, where the UEDS takes fused information of different urban sensor data, such as CDR, RF, transportation, social network and event-website data, local context and land use data, habitats mobility pattern, and their density distribution over time, sentiment and context information about an event extracted from social network and event blogs. Optimized fusion rules are required for 
the information fusion to assure the exact mixing of the information extracted from the datasets. Now, two possible match detection scenario is provided below:

1. Let, in a time interval $\Delta t$, two activities $E_{1}$ and $E_{2}$ from two users $x$ and $y$ stored in the same data source are considered matched, if and only if:

$$
\begin{gathered}
\Im\left(\mathrm{DS}_{E_{1}}^{x}\right)==\Im\left(\mathrm{DS}_{E_{2}}^{y}\right) \\
\left|t\left(\mathrm{DS}_{E_{1}}^{x}\right)-t\left(\mathrm{DS}_{E_{2}}^{y}\right)\right|<\Delta t
\end{gathered}
$$

where $\mathrm{DS}_{E_{m}}^{n}$ is the $m$-th activity of the $n$-th user stored in $\mathrm{DS}, \Im($.$) is the geo-location (i, j, t)$ of the event. Matching two datasets, $t($.$) is the time of the event.$

2. On the other hand, two activities $E_{1}$ and $E_{2}$ of two users $x$ and $y$ in different datasets (e.g., DS1 and DS2) are considered matched, if and only if:

$$
\begin{aligned}
& \Im_{d i s t}\left(\mathrm{DS} 1_{E_{1}}^{x}, \mathrm{DS} 2_{E_{2}}^{y}\right)<R \\
& t_{d i s t}\left(\mathrm{DS} 1_{E_{1}}^{x}, \mathrm{DS} 2_{E_{2}}^{y}\right)<\Delta t
\end{aligned}
$$

where $\left.\Im_{\text {dist }}\left({ }_{-},\right)_{-}\right)$and $t_{\text {dist }}\left({ }_{-},{ }_{-}\right)$are spatial and temporal distance respectively, and $R$ is the minimum distance coverage.

Given that a non-redundant dataset is available at hand for urban event detection, unusual over-crowd $\left(C_{o c}(i, j, t)\right)$ or under-crowd $\left(C_{u c}(i, j, t)\right)$ are detected by comparing the estimated or detected crowd size $\left(C_{e}(i, j, t)\right)$ to specific defined thresholds for a given scenario. These threshold values depend on local context information, and experts' experience and intuition which usually are different from each other. Thus there is no hard rule to select the value of this threshold [51] For example, the threshold value for over-crowd $\left(t h r_{o c}\right)$ can be 90-th percentile, and for under-crowd $\left(t h r_{u c}\right)$ can be the median of the dataset.

Therefore, $C_{o c}(i, j, t)$ is detected (the Red-shaded area in Figure 16), when $C_{e}(i, j, t)>t h r_{o c}$; and $C_{u c}(i, j, t)$ is detected (the Green-shaded area in Figure 16), when $C_{e}(i, j, t)<$ $t h r_{u c}$. This detected unusual over-crowd or under-crowd by the UEDS can then be mapped to an urban event at a geolocation $(i, j)$ as show in Figure 15 and be used by specific UAs to deliver personalized services.

\section{B. Decision Support System}

DSS can change the existing services adaptively based on the detected changes in the urban events. For example, in case of a transportation planning and management system, the transportation services might be modified based on the detected crowd events. As seen in Figure 16, the transportation services might be increased when $C_{o c}(i, j, t)$ is detected and decreased $C_{u c}(i, j, t)$ is detected. In addition, other transportation data, e.g., automated vehicle location [98] and arrival timing along with passenger waiting time [97] can be used to derive other DSS which may also be applied to provide modified transportation services that include rescheduling transport mobility of the usual trips.

\section{Open Challenges AND RESEARCh Opportunities}

Despite the work reported in the literature, there remain several challenging problems yet to be addressed. The following subsections outline few such challenges and possible underlying research opportunities therein.

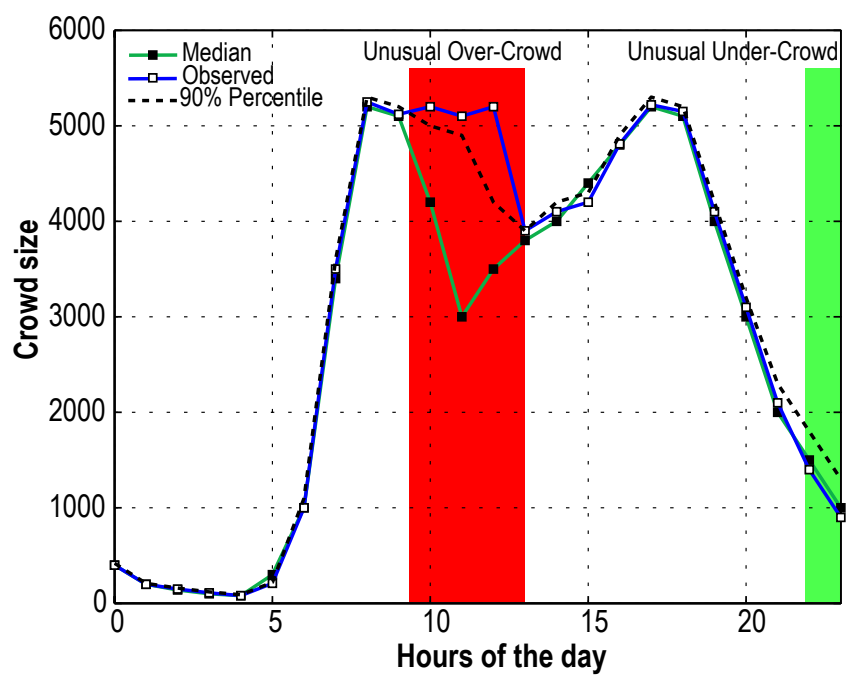

Fig. 16. Average hourly crowd size. The black dashed line shows 90-th percentile of the data, green line with filled box shows the observed data, and blue line with open box shows the median of the data in a day. The red-shaded area indicates the detected unusual over-crowd when the observed value is higher than 90-th percentile. On the other hand, green-shaded area indicates the detected unusual under-crowd when the observed value is lower than the median of the data.

\section{A. Uncertain and Noisy Data}

Urban sensing data are enormous in volumes and generated relentlessly. Almost all the urban sensors are prone to generate imprecise, incomplete, uncertain, and noisy data. Examples of such irregularities in data include: GPS data containing inherent measurement noise; RFID and Bluetooth data may generate high bit error rate during poor Signal-to-Noise Ratio and link failures; discrete CDR data due to switching between activity and non-activity modes. In addition, spatio-temporal bias can also generate uncertain data

Though different methods can be applied to handle incomplete and uncertain data, e.g., Kalman filter to estimate the state of dynamic system to filter noisy data; regression and interpolation to handle missing data via exploration; dynamic Bayesian network to handle the uncertain and noisy data, yet, the management and processing of such uncertain, noisy, and incomplete data remain an open challenge.

\section{B. Data Verification and Integrity}

In order to get the microscopic details of inhabitants, an urban planner collects city data generated by different sensors (e.g., Smart Card, Parking Lot Occupancy Sensor, etc.) or methods (e.g., census, survey, etc.) at different times. There are also self-reported data (i.e., WiFi), network recorded data (i.e., CDR), and social network data. Since these data are collected at different contexts and times, it is hard to substantiate the results obtained using a single dataset without crossverification for data integrity which is still an open challenge.

\section{Fusion and Fusion Rule}

Crowd event detection is context oriented and all the datasets may not always dispense comprehensive information 
about the crowd event [22]. For example, the spatial accuracy of a geo-location is necessary to identify a user's trajectory or the modes of transportation being used. Some of the data (e.g., CDR, social network data, etc.) has low spatial resolution whereas some data are incomplete as these are user-driven or participatory (e.g., WiFi). Therefore, to have amalgamated information from multiple datasets (e.g., CDR, WiFi, video, and social network data), information fusion can be applied. The fusion process is complex owing to data registration and alignment, conflicting and inconsistence of multiple datasets as well as their variable spatio-temporal resolution. Moreover, the fusion rules are context and problem specific. Thus, selecting optimal fusion rule to extract complementary information from different datasets for a certain application is also a challenge.

\section{Real-time Data Collection and Processing}

Data collected from different urban sensors at real-time are used for specific urban sensing applications, includingcrowd event detection, traffic monitoring, and transportation planning. Data generated from some of the sources such as video surveillance cameras and mobile activity (e.g., CDR) are massive. These data can be stored in real-time, but can not be processed. Though some real-time streaming platforms (such as Apache Spark, Apache Storm, Apache Samza, Amazon Kinesis, Microsoft StreamInsight, IBM InfoSphere Streams, and Informatica Vibe Data Stream, etc.) aim to process such data and generate information in near-real-time [135], [136], [137], [138], yet, the necessity of ad-hoc algorithms to process the data in real-time is still an open challenge.

\section{E. Data Privacy and Anonymity}

Not all data sources used for urban event detection are participatory or user driven, which may raise privacy concerns. For example, the trajectory data provided by CDR tracks individuals and preferences risking their privacy as it is possible to uniquely track $95 \%$ of peoples' trajectories by knowing only four spatio-temporal points [139]. Though various methods, e.g., obfuscation [140], $k$-anonymity [141], differentially private model [71], [142], information fusion and aggregation [143], have been proposed, yet privacy protection remains an open challenge.

\section{F. Novel Analysis Paradigms}

Recently, advanced machine learning techniques (e.g., Deep Learning or DL, Reinforcement Learning or RL) have successfully been employed in many applications including pattern recognition, big data analytics, etc. due to its capability to learn a problem at multiple levels using multiple features and abstractions and by interacting with the environment. DL has been successfully applied to- predict spatio-temporal traffic flow from big transport data [144], count people in extremely dense crowd [145], select efficient transportation based on human mobility [146], predict crowd flow in a city [147]. In addition, deep RL has also been applied in road transport support system [148]. Thus, development of novel machine learning techniques would provide solutions to detect crowd events using multiple datasets as discussed in Section II.

\section{CONCLUSION}

The growing usage of ubiquitous computing, supported by the massive development of ICT infrastructures, has become an inseparable part of everyday life. Duly, human activities are generating an enormous amount of data, describing the urban dynamics. This paper surveyed heterogeneous ubiquitous urban applications, the data produced by these applications, different application programming interfaces, benchmark crowd datasets, and various data generation and processing methods for the crowd event detection to demonstrate the relative merits and capabilities. Finally, existing open challenges, and forecasted research directions that may impact enhancement of the crowd event detection accuracy, have been outlined including an investigation into the critical issues, merits and capabilities of various existing contributions to smart transportation management and planning.

\section{ACKNOWLEDGMENT}

Financial support from the Erasmus Mundus - Action 2 Programme of the European Commission ('SmartLink', contract no. 552077) is kindly acknowledged.

\section{REFERENCES}

[1] M.-P. Pelletier, M. Trepanier, and C. Morency, "Smart card data use in public transit: A literature review," Transp. Res. Part C Emerg. Technol., vol. 19, no. 4, pp. 557-568, 2011.

[2] N. Nassir, M. Hickman, and Z. L. Ma, "Activity detection and transfer identification for public transit fare card data," Transportation, vol. 42 , no. 4, pp. 683-705, 2015

[3] D. Olgun, B. N. Waber, T. Kim, A. Mohan, K. Ara, and A. Pentland, "Sensible organizations: Technology and methodology for automatically measuring organizational behavior," IEEE Trans. Syst. Man. Cybern. B, vol. 39, no. 1, pp. 43-55, 2009.

[4] S. Colak, L. P. Alexander, B. G. Alvim, and M. C. Gonzalez, "Analyzing cell phone location data for urban travel: current methods, limitations, and opportunities," J. Transp. Res. Board, no. 2526, p. 126135, 2014.

[5] Y. Dong, F. Pinelli, Y. Gkoufas, Z. Nabi, F. Calabrese, and N. V. Chawla, "Inferring unusual crowd events from mobile phone call detail records," in Proc. ECML PKDD, 2015, pp. 474-492.

[6] J. Weppner and P. Lukowicz, "Bluetooth based collaborative crowd density estimation with mobile phones," in Proc. IEEE Int. Conf. Perv. Comput. Commun., 2013, pp. 193-200.

[7] N. Eagle and A. Pentland, "Reality mining: Sensing complex social systems," Pers. Ubiquit. Comput., vol. 10, no. 4, pp. 255-268, 2006.

[8] V. Kostakos, T. Camacho, and C. Mantero, "Towards proximity-based passenger sensing on public transport buses," Pers. Ubiquit. Comput., vol. 17, no. 8, pp. 1807-1816, 2013.

[9] M. Versichele, T. Neutens, M. Delafontaine, and N. Van de Weghe, "The use of bluetooth for analysing spatiotemporal dynamics of human movement at mass events: A case study of the ghent festivities," Applied Geography, vol. 32, no. 2, pp. 208-220, 2012.

[10] G. Michau, A. Nantes, A. Bhaskar, E. Chung, P. Abry, and P. Borgnat, "Bluetooth data in an urban context: Retrieving vehicle trajectories," IEEE Trans. Intell. Transp. Syst., vol. PP, no. 99, pp. 1-10, 2017.

[11] J. Weppner, B. Bischke, and P. Lukowicz, "Monitoring crowd condition in public spaces by tracking mobile consumer devices with wifi interface," in Proc. ACM Int. Jt. Conf. Perv. Ubiquit. Comput., 2016, pp. 1363-1371.

[12] C. Zhu, H. Zhu, Y. Ge, E. Chen, and Q. Liu, "Tracking the evolution of social emotions: A time-aware topic modeling perspective," in Proc. IEEE Int. Conf. Data Mining, 2015, pp. 517-544.

[13] W. Xi, J. Zhao, X. Y. Li, K. Zhao, S. Tang, X. Liu, and Z. Jiang, "Electronic frog eye: Counting crowd using wifi," in Proc. IEEE INFOCOM, 2014, pp. 361-369.

[14] N. Koshak and A. Fouda, "Analyzing pedestrian movement in mataf using gps and gis to support space redesign," in Proc. 9th Int. Conf. Des. Decis. Support Syst. Archit. Urban Plan., no. July, 2008, pp. 1-14. 
[15] F. Calabrese, C. Ratti, M. Colonna, P. Lovisolo, and D. Parata, "Rome real-time urban monitoring using cell phones: A case study," IEEE Trans. Intell. Transp. Syst., vol. 12, no. 1, pp. 141-151, 2011.

[16] Y. Dong, F. Pinelli, Y. Gkoufas, Z. Nabi, F. Calabrese, and et al., "Inferring unusual crowd events from mobile phone call detail records," in Proc. ECML PKDD, vol. 9285, 2015, pp. 474-492.

[17] H. Yang, K. Ozbay, and B. Bartin, "Enhancing the quality of infraredbased automatic pedestrian sensor data by nonparametric statistical method," Transport. Res. Rec., vol. 2264, pp. 11-17, 2011.

[18] M. Rodriguez, J. Sivic, I. Laptev, and J. Y. Audibert, "Data-driven crowd analysis in videos," in Proc. ICCV, 2011, pp. 1235-1242.

19] Z. Wang, H. Liu, Y. Qian, and T. Xu, "Crowd density estimation based on local binary pattern co-occurrence matrix," Proc. IEEE Int. Conf. Multimedia Expo. Workshops, pp. 372-377, 2012.

[20] T. Li, H. Chang, M. Wang, B. Ni, R. Hong, and S. Yan, "Crowded scene analysis: A survey," IEEE Trans. Circuits Syst. Video Technol., vol. 25 , no. 3 , pp. $367-386,2014$

[21] A. Candelieri and F. Archetti, "Detecting events \& sentiment on twitte for improving urban mobility," in Proc. ESSEM, 2015, pp. 106-15.

[22] F. Botta, H. S. Moat, and T. Preis, "Quantifying crowd size with mobile phone and twitter data," Royal Soc. Open Sci., pp. 1-6, 2015.

[23] Z. Z. Alp and S. G. Oduducu, "Extracting topical information of tweets using hashtags," in Proc. 2015 ICMLA, 2015, pp. 644-648.

[24] F. Calabrese, L. Ferrari, and V. D. Blondel, "Urban sensing using mobile phone network data: A survey of research," ACM Comput. Surv. vol. 47 , no. 2 , pp. $25: 1-25: 20,2014$

[25] Z. Zhao, J. Zhao, and H. N. Koutsopoulos, "Individual-level trip detection using sparse call detail record data based on supervised statistical learning," in Proc. Transp. Res. Board 95th Annu. Meet., 2016, pp. 1-18

[26] Y. Wang, D. Zhang, L. Hu, Y. Yang, and L. H. Lee, "A data-driven and optimal bus scheduling model with time-dependent traffic and demand," IEEE Trans. Intell. Transp. Syst., vol. PP, no. 99, pp. 1-10, 2017

[27] Dadax. (2012) Worldometers. Accessed on 2017-01-02. [Online]. Available: www.worldometers.info/world-population/

[28] Statista. (2008) Number of mobile phone users worldwide from 2013 to 2019 (in billions). Accessed on 2017-07-07. [Online]. Available: https://goo.gl/Twx8RK

[29] S. Jiang, Y. Yang, G. Fiore, J. Ferreira, E. Frazzoli, and M. C. Gonzalez, "A review of urban computing for mobile phone traces: Current methods, challenges and opportunities," in Proc. UrbComp, 2013, pp. 2:1-2:9.

[30] A. Janecek, K. A. Hummel, D. Valerio, F. Ricciato, and H. Hlavacs, "Cellular data meet vehicular traffic theory: Location area updates and cell transitions for travel time estimation," in Proc. ACM Conf. Ubiquit. Comput., 2012, pp. 361-370.

[31] V. Frias-Martinez, Y. Moumni, and E. Frias-Martinez, "Estimation of traffic flow using passive cell-phone data," in Proc. Intl. Workshop Data Sci. Macro-Modeling, 2014, pp. 13:1-13:2.

[32] M. C. Gonzalez, C. A. Hidalgo, and A.-L. Barabasi, "Understanding individual human mobility patterns," Nature, vol. 453, no. 7196, pp. 779-782, 2008

[33] A. Janecek, D. Valerio, K. A. Hummel, F. Ricciato, and H. Hlavacs, "The cellular network as a sensor: From mobile phone data to realtime road traffic monitoring," IEEE Trans. Intell. Transp. Syst., vol. 16 no. 5, pp. 2551-2572, 2015.

[34] L. Schauer, M. Werner, and P. Marcus, "Estimating crowd densities and pedestrian flows using wi-fi and bluetooth," in Proc. 11th Int. Conf. Mob. Ubiq. Syst., 2014, pp. 171-177.

[35] J. C. S. Jacques, S. R. Mussef, and C. R. Jung, "Crowd analysis using computer vision techniques," IEEE Signal Process. Mag., vol. 27, no. 5 , pp. 66-77, 2010

[36] B. Zhan, D. N. Monekosso, P. Remagnino, S. A. Velastin, and L.-Q Xu, "Crowd analysis: a survey," Mach. Vis. Appl., vol. 19, no. 5, pp. 345-357, 2008

[37] S. A. M. Saleh, S. A. Suandi, and H. Ibrahim, "Recent survey on crowd density estimation and counting for visual surveillance," Eng. Appl. Artif. Intell., vol. 41, pp. 103-114, 2015.

[38] H. Saif, M. Fernandez, Y. He, and H. Alani, "Evaluation datasets for twitter sentiment analysis: A survey and a new dataset, the sts-gold," in Proc. ESSEM, 2013, pp. 9-21.

[39] B. Batrinca and P. C. Treleaven, "Social media analytics: a survey of techniques, tools and platforms," AI \& Society, vol. 30, no. 1, pp. 89-116, 2015.

[40] M. Adedoyin-Olowe, M. M. Gaber, and F. T. Stahl, "A survey of data mining techniques for social media analysis," J. Data Mining \& Digit. Humanit., vol. 2014, 2014
[41] L. Jin, Y. Chen, T. Wang, P. Hui, and A. V. Vasilakos, "Understanding user behavior in online social networks: a survey," IEEE Commun. Mag., vol. 51, no. 9, pp. 144-150, 2013.

[42] A. Gal-Tzur, S. M. Grant-Muller, E. Minkov, and S. Nocera, "The impact of social media usage on transport policy: Issues, challenges and recommendations," Procedia Soc. Behav. Sci., vol. 111, pp. 937946, 2014.

[43] D. Efthymiou and C. Antoniou, "Use of social media for transport data collection," Procedia Soc. Behav. Sci., vol. 48, pp. 775-785, 2012.

[44] S. M. Grant-Muller, A. Gal-Tzur, E. Minkov, S. Nocera, T. Kuflik, and I. Shoor, "Enhancing transport data collection through social media sources: methods, challenges and opportunities for textual data," IET Intell. Transp. Syst., vol. 9, no. 4, pp. 407-417, 2015

[45] Y. Liang, J. Caverlee, Z. Cheng, and K. Y. Kamath, "How big is the crowd?: event and location based population modeling in social media," in Proc. HT, 2013, pp. 99-108

[46] R. O. Sinnott and W. Chen, "Estimating crowd sizes through social media," in Proc. PerCom Workshops, 2016, pp. 1-6.

[47] M. S. Bernstein, E. Bakshy, M. Burke, and B. Karrer, "Quantifying the invisible audience in social networks," in Proc. CHI, 2013, pp. 21-30.

[48] T. Ma, G. Motta, and K. Liu, "Delivering real-time information service on public transit: A framework," IEEE Trans. Intell. Transp. Syst., vol. PP, no. 99, pp. 1-15, 2017.

[49] V. A. Traag, A. Browet, F. Calabrese, and F. Morlot, "Social event detection in massive mobile phone data using probabilistic location inference," in Proc. SocialCom/PASSAT, 2011, pp. 625-628.

[50] Y. Mowafi, A. Zmily, D. e. D. Abou-Tair, and D. Abu-Saymeh, "Tracking human mobility at mass gathering events using wisp," in Proc. FGCT, 2013, pp. 157-162.

[51] F. C. Pereira, F. Rodrigues, E. Polisciuc, and M. Ben-Akiva, "Why so many people? explaining nonhabitual transport overcrowding with internet data," IEEE Trans. Intell. Transp. Syst., vol. 16, no. 3, pp. 1370-1379, 2015

[52] A. Tirachini, D. A. Hensher, and J. M. Rose, "Crowding in public transport systems: Effects on users, operation and implications for the estimation of demand," Transp. Res. Pt. A-Policy Pract., vol. 53, pp. 36-52, 2013

[53] C. M. Schneider, V. Belik, T. Couronne, Z. Smoreda, and M. C. Gonzalez, "Unravelling daily human mobility motifs," J. R. Soc. Interface, vol. 10, no. 84, p. 20130246, 2013

[54] R. Sharma and T. Guha, "A trajectory clustering approach to crowd flow segmentation in videos," in Proc. ICIP, 2016, pp. 1200-1204.

[55] S. A. H. AlGadhi, H. S. Mahmassani, and R. Herman, "A speedconcentration relation for bi-directional crowd movements with strong interaction," in Pedestrian and evacuation dynamics, M. Schreckenberg and S. Deo-Sarma, Eds. Springer, 2002, ch. 1, pp. 3-20.

[56] S. Hoogendoorn and P. Bovy, "Pedestrian route-choice and activity scheduling theory and models," Transport. Res. Part B, vol. 38, no. 2, pp. 169-190, 2004

[57] S. P. Hoogendoorn and W. Daamen, "Pedestrian behavior at bottlenecks," Transport. Sci., vol. 39, no. 2, pp. 147-159, 2005.

[58] S. Bandini, A. Gorrini, and G. Vizzari, "Towards an integrated approach to crowd analysis and crowd synthesis: A case study and first results," Pattern Recogn. Lett., vol. 44, pp. 16-29, 2014.

[59] V. J. Kok, M. K. Lim, and C. S. Chan, "Crowd behavior analysis: A review where physics meets biology," Neurocomputing, vol. 177, pp. 342-362, 2016

[60] M. S. Zitouni, H. Bhaskar, J. Dias, and M. E. Al-Mualla, "Advances and trends in visual crowd analysis: A systematic survey and evaluation of crowd modelling techniques," Neurocomputing, vol. 186, pp. 139-59, 2016.

[61] S. R. Musse and D. Thalmann, "A model of human crowd behavior Group inter-relationship and collision detection analysis," in Proc. Eurographics, 1997, pp. 39-51.

[62] V. Price, Public Opinion, ser. Communication Concepts. California, USA: Sage Publications, 1992.

[63] M. Khan and K. Han, "A survey of context aware vertical handover management schemes in heterogeneous wireless networks," Wirel. Pers. Commun., vol. 85, no. 4, pp. 2273-2293, 2015.

[64] J. L. Toole, S. Colak, B. Sturt, L. P. Alexander, A. Evsukoff, and M. C. Gonzlez, "The path most traveled: Travel demand estimation using big data resources," Transp. Res. Part C Emerg. Technol., vol. 58, pp. 162 177, 2015.

[65] S. Isaacman, R. Becker, R. Caceres, S. Kobourov, J. Rowland, and A. Varshavsky, "A tale of two cities," in Proc. 11th Workshop Mob. Comp. Sys. Appl., 2010, pp. 19-24. 
[66] S. V. D. Elzen, J. Blaas, D. Holten, J.-k. Buenen, J. J. V. Wijk, and et al., "Exploration and analysis of massive mobile phone data: A layered visual analytics approach," in Proc. NetMob, 2013, pp. 1-10.

[67] T. Louail, M. Lenormand, O. G. C. Ros, M. Picornell, R. Herranz, E. Frias-Martinez, J. J. Ramasco, and M. Barthelemy, "From mobile phone data to the spatial structure of cities," Sci. Rep., vol. 4, p. srep05276, 2014

[68] S. Isaacman, R. Becker, R. Caceres, S. Kobourov, M. Martonosi, J. Rowland, and A. Varshavsky, "Identifying important places in people's lives from cellular network data," in Proc. Pervasive, 2011, pp. 133-151.

[69] G. Di Lorenzo, M. Sbodio, F. Calabrese, M. Berlingerio, F. Pinelli, and R. Nair, "Allaboard: Visual exploration of cellphone mobility data to optimise public transport," IEEE Trans. Visual. Comput. Grap., vol. 22, no. 2, pp. 1036-1050, 2016.

[70] F. Girardin, A. Vaccari, A. Gerber, A. Biderman, and C. Ratti, "Quantifying urban attractiveness from the distribution and density of digital footprints," IJSDIR, vol. 4, pp. 175-200, 2009.

[71] D. J. Mir, S. Isaacman, R. Caceres, M. Martonosi, and R. N. Wright, "DP-WHERE: Differentially private modeling of human mobility," in Proc. BigData, 2013, pp. 580-588.

172] F. H. Z. Xavier, L. M. Silveira, J. M. Almeida, A. Ziviani, C. H. S Malab, and et al., "Understanding human mobility due to large-scale events," in Proc. NetMob, 2013, pp. 1-3.

[73] V. Kostakos, T. Camacho, and C. Mantero, "Wireless detection of endto-end passenger trips on public transport buses," in Proc. ITSC, 2010, pp. $1795-1800$

[74] M. S. Frumin, "Automatic data for applied railway management passenger demand, service quality measurement, and tactical planning on the london overground network," PhD Thesis, MIT, 2010.

[75] X. Shi, Q. Zhou, X. Qu, G. Liu, and Z. Gong, "Understanding city dynamics based on public bicycle data: A case study in Hangzhou," in Proc. SKIMA, 2016, pp. 146-150.

[76] P. Arcaini, G. Bordogna, D. Ienco, and S. Sterlacchini, "User-driven geo-temporal density-based exploration of periodic and not periodic events reported in social networks," Informa. Sci., vol. 341, pp. 122 $143,2016$.

77] Z. Xu, Y. Liu, N. Yen, L. Mei, X. Luo, and et al., "Crowdsourcing based description of urban emergency events using social media big data," IEEE Trans. Cloud Comput., vol. PP, 2016.

[78] M. Khalifa, R. P. Diaz Redondo, A. F. Vilas, and S. S. Rodriguez, "Identifying urban crowds using geo-located social media data: a twitter experiment in new york city," J. Intell. Inform. Syst., pp. 1-22, 2016.

[79] A. Guille and C. Favre, "Event detection, tracking, and visualization in Twitter: a mention-anomaly-based approach," Soc. Netw. Anal. Min., vol. 5, no. 1, p. 18, 2015.

[80] C. C. Loy, K. Chen, S. Gong, and T. Xiang, "Crowd counting and profiling: Methodology and evaluation," in Modeling, Simulation and Visual Analysis of Crowds: A Multidisciplinary Perspective, 2013, pp. 347-382.

[81] S. Yi, X. Wang, C. Lu, J. Jia, and H. Li, "lo regularized stationarytime estimation for crowd analysis," IEEE Trans. Pattern Anal. Mach Intell., vol. 39, no. 5, pp. 981-994, 2017.

[82] L. Boominathan, S. S. S. Kruthiventi, and R. V. Babu, "Crowdnet: A deep convolutional network for dense crowd counting," in Proc. ACM Multimedia Conf., 2016, pp. 640-644.

[83] A. C. Davies, J. H. Yin, and S. Velastin, "Crowd monitoring using image processing," Electron. Commun. Eng. J., vol. 7, no. 1, pp. $37-$ 47, 1995.

[84] S.-Y. Cho and T. W. S. Chow, "A fast neural learning vision system for crowd estimation at underground stations platform," Neural Process. Lett., vol. 10, no. 2, pp. 111-120, 1999.

[85] R. Ma, L. Li, W. Huang, and Q. Tian, "On pixel count based crowd density estimation for visual surveillance," in Proc. ICCIS, 2004, pp. $170-173$

[86] N. Hussain, H. S. M. Yatim, N. L. Hussain, J. L. S. Yan, and F. Haron, "CDES: A pixel-based crowd density estimation system for masjid alharam," Safety Science, vol. 49, no. 6, pp. 824-833, 2011.

[87] A. Albiol, M. J. Silla, A. Albiol, and J. M. Mossi, "Video analysis using corner motion statistics," in Proc. CVPR, 2009, pp. 31-37.

[88] B. Sirmacek and P. Reinartz, "Automatic crowd analysis from very high resolution satellite images," Int. Arch. Photogramm. Remote Sens. Spatial Inf. Sci., vol. XXXVIII-3/W22, pp. 221-226, 2011.

[89] O. Meynberg, S. Cui, and P. Reinartz, "Detection of high-density crowds in aerial images using texture classification," Remote Sensing, vol. 8 , no. 6 , p. 470,2016
[90] M. Wirz, P. Schlapfer, M. B. Kjargaard, D. Roggen, S. Feese, and G. Troster, "Towards an online detection of pedestrian flocks in urban canyons by smoothed spatio-temporal clustering of gps trajectories,' Proc. LBSN, pp. 17-24, 2011.

[91] S. B. Ranneries, M. E. Kalor, S. A. Nielsen, L. N. Dalgaard, and et al., "Wisdom of the local crowd: detecting local events using social media data," in Proc. WebSci, 2016, pp. 352-354.

[92] A. Morrison, M. Bell, and M. Chalmers, "Visualisation of spectator activity at stadium events," in Proc. IV, 2009, pp. 219-226.

[93] C. Xu, B. Firner, R. S. Moore, Y. Zhang, and et al., "SCPL: Indoor device-free multi-subject counting and localization using radio signal strength," in Proc. IPSN, 2013, pp. 79-90.

[94] Y. Fukuzaki, M. Mochizuki, K. Murao, and N. Nishio, "A pedestrian flow analysis system using wifi packet sensors to a real environment," in Proc. UbiComp, 2014, pp. 721-730.

[95] Y. Yuan, J. Zhao, C. Qiu, and W. Xi, "Estimating crowd density in an rf-based dynamic environment," IEEE Sens. J., vol. 13, no. 10, pp. 3837-3845, 2013.

[96] W. Zeng, C. W. Fu, S. M. Arisona, S. Schubiger, R. Burkhard, and K. L. Ma, "Visualizing the relationship between human mobility and points of interest," IEEE Trans. Intell. Transp. Syst., pp. 1-14, 2017.

[97] F. McLeod, "Estimating bus passenger waiting times from incomplete bus arrivals data," J. Oper. Res. Soc., vol. 58, no. 11, pp. 1518-1525, 2007

[98] B. Barabino, M. Di Francesco, and S. Mozzoni, "Time reliability measures in bus transport services from the accurate use of automatic vehicle location raw data," Qual. Reliab. Eng. Int., vol. 33, no. 5, pp. 969-978, 2017

[99] X. Han, Y. Fu, Z. Qin, and K. Xing, "An automatic method of building extraction from high resolution satellite images," 2009, p. 749540.

[100] S. Penmetsa, F. Minhuj, A. Singh, and S. Omkar, "Autonomous uav for suspicious action detection using pictorial human pose estimation and classification," Electron. Lett. Comput. Vis. Image Anal., vol. 13 no. 1, pp. 18-32, 2014

[101] G. Salvo, L. Caruso, and A. Scordo, "Urban traffic analysis through an UAV," Procedia Soc. Behav. Sci., vol. 111, pp. 1083-1091, 2014.

[102] Y. De Mulder, G. Danezis, L. Batina, and B. Preneel, "Identification via location-profiling in gsm networks," in Proc. WPES, 2008, pp. 23-32.

[103] K. Still, "Crowd dynamics," PhD Thesis, University of Warwick, 2000

[104] Z. Fang, J. P. Yuan, Y. C. Wang, and S. M. Lo, "Survey of pedestrian movement and development of a crowd dynamics model," Fire Safety J., vol. 43, no. 6, pp. 459-465, 2008.

[105] T. Zhao, R. Nevatia, and B. Wu, "Segmentation and tracking of multiple humans in crowded environments," IEEE Trans. Pattern Anal. Mach. Intell., vol. 30, no. 7, pp. 1198-1211, 2008.

[106] P. Kohli, J. Rihan, M. Bray, and P. H. S. Torr, "Simultaneous segmentation and pose estimation of humans using dynamic graph cuts," Int. J. Comput. Vis., vol. 79, no. 3, pp. 285-298, 2008.

[107] G. J. Brostow and R. Cipolla, "Unsupervised bayesian detection of independent motion in crowds," in Proc. CVPR, 2006, pp. 594-601.

[108] E. F. Nakamura, A. A. F. Loureiro, and A. C. Frery, "Information fusion for wireless sensor networks: Methods, models, and classifications," ACM Comput. Surv., vol. 39, no. 3, 2007.

[109] F. Castanedo, "A review of data fusion techniques," Scientific World J., vol. 2013, pp. 1-19, 2013.

[110] M. Mahbub, S. M. R. Ali, Z. Iqbal, M. S. Kaiser, and M. S. Islam, "Performance analysis of cognitive cooperative communications for wban," in Proc. ICTP, 2015, pp. 1-5.

[111] V. D. B. et. al., "Data for development: the D4D challenge on mobile phone data," CoRR, vol. abs/1210.0137, 2012.

[112] A. McDiarmid. (2011) Nodobo data. Accessed on 2016-11-02. [Online]. Available: http://nodobo.com/release.html

[113] H. Idrees, I. Saleemi, C. Seibert, and M. Shah. (2016, Nov) Multi-source multi-scale counting in extremely dense crowd images. Accessed on 2017-07-07. [Online]. Available: http://crcv.ucf.edu/ projects/crowdCounting/index.php

[114] D. Ryan and S. Denman. (2012) Crowd counting database. Accessed on 2017-07-27. [Online]. Available: https://researchdata.ands.org.au/ crowd-counting-database/448416

[115] U.S. Department of Transportation. (2005) Traffic analysis tools program. Accessed on 2016-11-02. [Online]. Available: http://ops. fhwa.dot.gov/trafficanalysistools/ngsim.htm

[116] J. Shao, C. C. Loy, and X. Wang. (2014) Cuhk crowd dataset. Accessed on 2016-11-02. [Online]. Available: www.ee.cuhk.edu.hk/ $\sim$ xgwang/datasets.html 
[117] M. Jiang, J. Xu, and Q. Zhao. (2014) Eyecrowd dataset. Accessed on 2017-07-07. [Online]. Available: www-users.cs.umn.edu/ qzhao/ crowd.html

[118] US Government. (2016) U.s. gov. open traffic data. Accessed on 201611-17. [Online]. Available: https://catalog.data.gov/dataset?q=traffic

[119] Government of Ireland. (2013) Irish transport data. Accessed on 2016-12-07. [Online]. Available: https://data.gov.ie/data/search? theme-primary=Transport

[120] Mayor of London. (2014) Transport for london (tfl). Accessed on 2016 11-02. [Online]. Available: https://data.london.gov.uk/publisher/tfl

[121] B. G. Matthias Mueller, Neil Smith. (2016, June) Uav 50 and uav123. Accessed on 2017-07-16. [Online]. Available: https: //ivul.kaust.edu.sa/Pages/pub-benchmark-simulator-uav.aspx

[122] Harvard Dataverse. (2015) A multi-source dataset of urban life in the city of milan and the province of trentino. Accessed on 2016-11-02. [Online]. Available: https://dataverse.harvard.edu/ dataverse/bigdatachallenge

[123] F. Botta, H. S. Moat, and T. Preis. (2015) Datasets for quantifying crowd size with mobile phone and Twitter data. Accessed on 2016-11-02. [Online]. Available: http://datadryad.org/resource/doi: 10.5061/dryad.1rk60

[124] City of Cambridge. (2015) Cambridgema open data. Accessed on 2016-11-17. [Online]. Available: https://data.cambridgema.gov/

[125] CMAP. (2007) Travel tracker survey - cmap. Accessed on 201701-02. [Online]. Available: www.cmap.illinois.gov/data/transportation/ travel-tracker-survey

[126] Google. (2006) Google trends. Accessed on 2016-11-17. [Online]. Available: www.google.com/trends/

[127] Mayor of London. (2015) Transport for london unified api. Accessed on 2016-11-17. [Online]. Available: https://api.tfl.gov.uk/

[128] Dandelin API. (2014) Open big data. Accessed on 2016-11-02. [Online]. Available: https://dandelion.eu/datamine/open-big-data/

[129] Twitter. (2014) REST APIs. Accessed on 2016-11-02. [Online]. Available: https://dev.twitter.com/rest/public

[130] Flickr. (2004) Flickr API. Accessed on 2016-11-12. [Online] Available: www.flickr.com/services/api/

[131] TransportAPI. (2016) Transportapi. Accessed on 2016-11-02. [Online]. Available: www.transportapi.com/

[132] Microsoft. (2010) Bing maps. Accessed on 2017-07-07. [Online]. Available: https://msdn.microsoft.com/en-us/library/ff428642.aspx

[133] Google. (2009, June) Google map api for every platform. Accessed on 2017-07-16. [Online]. Available: https://developers.google.com/maps/

[134] Atlassian Developers. (2016, May) Crowd rest api. Accessed on 2017-07-02. [Online]. Available: https://developer.atlassian.com/ display/CROWDDEV/Crowd+REST+APIs

[135] SQLstream Inc. (2005) Processing and analyzing streams of CDRs in real time. Accessed on 2016-11-02. [Online]. Available: https://goo.gl/XrZecj

[136] S. Shahrivari, "Beyond batch processing: Towards real-time and streaming big data," Computers, vol. 3, no. 4, pp. 117-129, 2014

[137] V. Agneeswaran, Big Data Analytics Beyond Hadoop: Real-Time Applications with Storm, Spark, and More Hadoop Alternatives, $1 \mathrm{st}$ ed. USA: Pearson FT Press, 2014.

[138] L. Lopez and S. Chai, "Streaming data movement for real-time image analysis," J. Signal Process. Syst., vol. 62, no. 1, pp. 29-42, 2011.

[139] Y. A. Montjoye, C. A. Hidalgo, M. Verleysen, and V. D. Blondel, "Unique in the crowd: The privacy bounds of human mobility," Sci. Rep., vol. 3, p. srep01376, 2013.

[140] J. Krumm, "A survey of computational location privacy," Pers. Ubiquit. Comput., vol. 13, no. 6, pp. 391-399, 2009.

[141] B. Gedik and L. Liu, "Protecting location privacy with personalized kanonymity: Architecture and algorithms," IEEE Trans. Mobile Comput. vol. 7, no. 1, pp. 1-18, 2008.

[142] H. Roy, M. Kantarcioglu, and L. Sweeney, "Practical differentially private modeling of human movement data," in Proc. DBSec, 2016 , pp. $170-178$.

[143] G. Navarro Arribas and V. Torra, "Information fusion in data privacy: A survey," Inf. Fusion, vol. 13, no. 4, pp. 235-244, 2012.

[144] Y. Lv, Y. Duan, W. Kang, Z. Li, and F. Y. Wang, "Traffic flow prediction with big data: A deep learning approach,” IEEE Trans. Intell. Transp. Syst., vol. 16, no. 2, pp. 865-873, 2015.

[145] C. Wang, H. Zhang, L. Yang, S. Liu, and X. Cao, "Deep people counting in extremely dense crowds," in Proc. MM, 2015, pp. 12991302 .

[146] X. Song, H. Kanasugi, and R. Shibasaki, "Deeptransport: Prediction and simulation of human mobility and transportation mode at citywide level," in Proc. IJCAI, 2016, pp. 2618-24.
[147] J. Zhang, Y. Zheng, D. Qi, R. Li, X. Yi, and T. Li, "Predicting citywide crowd flows using deep spatio-temporal residual networks," CoRR, vol. abs/1701.02543, 2017.

[148] W. Genders and S. Razavi, "Using a deep reinforcement learning agent for traffic signal control," CoRR, vol. abs/1611.01142, 2016.

Cite as: M.S. Kaiser, K.T. Lwin, M. Mahmud, D. Hajializadeh, T. Chaipimonplin, A. Sarhan, M.A. Hossain. (2018) Advances in Crowd Analysis for Urban Applications Through Urban Event Detection.

IEEE Transactions on Intelligent Transportation Systems. doi: 10.1109/TITS.2017.2771746

1524-9050 @ 2017 IEEE. Personal use is permitted, but republication/redistribution requires IEEE permission. 


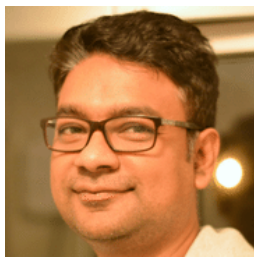

M Shamim Kaiser (M'07, SM'16) received the Bachelor and Master degrees in Applied Physics Electronics and Communication Engineering from the University of Dhaka, Bangladesh 2002 and 2004 respectively, and the Ph.D. degree in Telecommunications from Asian Institute of Technology (AIT) Pathumthani, Thailand in 2010. Currently, he is working as Associate Professor in the Institute of IT of Jahangirnagar University, Dhaka. His current research interests include Multi-hop cellular network; Cognitive radio network, Renewable Energy, Big Data analytics and Cyber Security. He has received 'Best Paper Awards' for three conference papers (COMSNETS 2010, ICEEICT 2014, ICEEICT 2015) as an author/co-author. Dr. Kaiser is a Life Member of Bangladesh Electronic Society and Bangladesh Physical Society. He is a senior member of IEEE, USA, a member of IEICE, Japan and Chapter Chair of IEEE Computer Society BD Chapter.

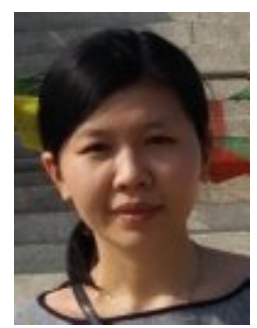

Khin T Lwin received a B.Sc. (Hons) and $\mathrm{PhD}$ degree in Computer Science from the University Of Nottingham, UK in 2010 and 2015 respectively. She also received the Springer Science and Business Media Prize, the University Prize for Academic Excellence and the International Research Excellence scholarship from Springer and University of Nottingham in 2010. She is currently a research fellow at the Anglia Ruskin IT Research Institute. She is a core member of the Big Data and Cyber Security research group. Her current research interests include computational intelligence, decision support systems, cyber security, portfolio optimization, big data analytics, machine learning, multi-objective optimization, evolutionary algorithms, heuristics and meta-heuristics.

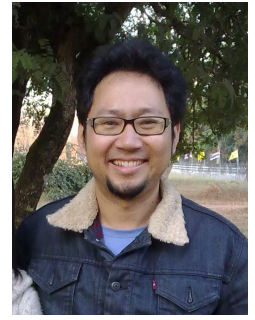

Tawee Chaipimonplin Tawee Chaipimonplin graduated in B.Sc. (Soil Science) from Khon Kaen University, Khon Kaen in 1997, Thailand, M.Ap.Sc (Geospatial Information) from RMIT University, Melbourne, Australia in 2002, Certificate of Proceeding the Radar Data of Earth Surface Remote Sensing from NPO Mashinostroyenia, Moscow, Russian Federation in 2004 and Ph.D. from the University of Leeds, UK in 2010 . He is a member of the Regional Center for Climate and Environmental Studies (RCCES) and an assistant professor at Department of Geography, Chiang Mai University. His research interest includes Artificial Neural Network (ANN), Geographic Information System (GIS) and Remote Sensing for natural hazard.

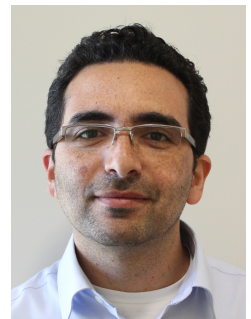

Ahmed Sarhan (M14) received the $\mathrm{PhD}$ degree from the Faculty of Engineering and Built Environment, University of Nottingham, UK, in 2012. He received the BSc degree in Architectural Engineering from Cairo University, Egypt, in 1998, and the MSc degree from the Department of Computer Science and IT, University of Nottingham, UK, in 2004. Dr. Sarhan is currently serving as a Senior Lecturer and the director of Virtualization, Simulation, and Infrastructure Research Group, in the Department of Computing \& Technology, Anglia Ruskin University, UK. Areas of teaching, research, and supervision include MultiAgent Systems and Agent Based Modelling, Data Mining and Warehousing (including Big Data), Smart Cities and Smart Homes, Decision Support Systems, Serious Games (Education and Simulation), and Inclusive Design tools. Dr Sarhan is currently a Fellow of Higher Education Academy.

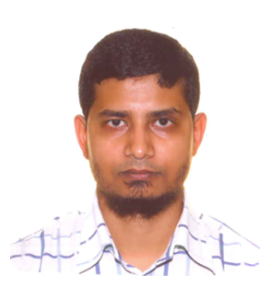

Mufti Mahmud (M'08, SM'16) received B.Sc. (University of Madras, 2001) and M.Sc. (University of Mysore, 2003) in Computer Science from India; and M.S. in Bionanotechnology (University of Trento, 2008) and Ph.D. in Bioengineering (University of Padova, 2011) from Italy. He was a recipient of Marie-Curie Fellowship. With over 60 publications in leading Journals and Conferences, Dr. Mahmud is an expert in analysis of brain signals $\mathrm{He}$ is the lead contributor of two open source toolboxes (SigMate, and QSpike Tools) for processing and analysis of extracellular neuronal signals. In addition, Dr. Mahmud's expertise includes BigData analysis, assistive brain-machine interfacing, computational neuroscience, personalized and preventive $[\mathrm{e} / \mathrm{m}]$-healthcare, and cloud-and-distributed computing. Dr. Mahmud serves as an Associate Editor of Springer's Cognitive Computation journal, successfully organized over 10 special sessions in leading conferences, served many reputed conferences in different capacities (e.g., programme, organization \& advisory committee member), and also served as referee for many journals.

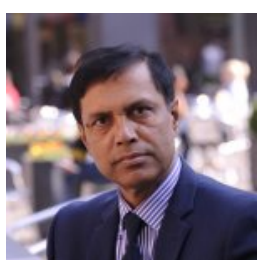

M A Hossain received his $\mathrm{PhD}$ degree in 1995 from the Department of Automatic Control and Systems Engineering, University of Sheffield, UK. $\mathrm{He}$ is currently serving as the Director of the Anglia Ruskin IT Research Institute at Anglia Ruskin University, Cambridge, UK. Before this, he also served in Northumbria University at Newcastle, University of Bradford, Sheffield University, Sheffield Hallam University and the University of Dhaka (as Chairman of the CSE). He has extensive research experience in computational intelligence, cyber security, decision support systems, digital diagnosis, real-time and adaptive algorithm design. Prof. Hossain has led many large EU funded projects as an International lead investigator, worth over 14 million. He acted as a conference/programme chair and IPC member of over 30 international conferences. With a publication in Nature, he has published over 275 refereed research articles, contributed in 12 books, received the "F C Williams 1996" award for an IET Journal and 'Best Paper Awards' for five conference papers (CSBio2010, IEEE CIS2012, SKIMA 2012, PACBB 2013 and SKIMA 2014) as an author/co-author.

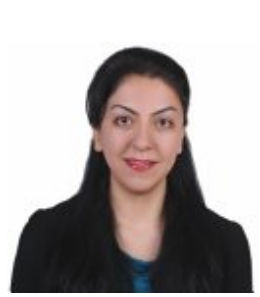

Donya Hajializadeh is a senior lecturer and a chartered engineer with 6 years of experience in innovative research and over 3 years of experience in HE teaching. Following her Masters, Donya competed a $\mathrm{PhD}$ entitled Combined Probabilistic Modelling of Load and Resistance in Bridges funded by the EU FP7. Donya joined RODIS in October 2013 as a Research Engineer and worked on several research projects funded by the EU FP7 such as: BridgeMon, RAIN, DESTinationRAIL. Donya also worked on different projects for the Irish National Road Authority and the Conference of European Directors of Roads. In June 2016, Donya joined Anglia Ruskin University's Engineering and the Built Environment Department as a Senior Lecturer. Currently, she is involved in several research studies related to Smart Cities. 